\title{
Arithmetic hypergeometric series
}

\author{
W. Zudilin
}

\begin{abstract}
The main goal of our survey is to give common characteristics of auxiliary hypergeometric functions (and their generalisations), functions which occur in number-theoretical problems. Originally designed as a tool for solving these problems, the hypergeometric series have become a connecting link between different areas of number theory and mathematics in general.
\end{abstract}

Bibliography: 183 titles.

Keywords: hypergeometric series; zeta value; diophantine approximation; irrationality measure; modular form; Ramanujan's mathematics; Calabi-Yau differential equation; Mahler measure; Wilf-Zeilberger theory; algorithm of creative telescoping.

\section{Contents}

Introduction 2

1. Arithmetic of the values of Riemann's zeta function 6

1.1. Apéry's theorem 6

1.2. Hypergeometric series and multiple integrals 11

1.3. Simultaneous approximations to $\zeta(2)$ and $\zeta(3) \quad 15$

1.4. $q$-Analogues of zeta values 18

1.5. Lower bound for $\left\|(3 / 2)^{k}\right\|$ and Waring's problem 21

2. Calabi-Yau differential equations 22

2.1. Arithmetic differential equations of order 2 and $3 \quad 22$

2.2. Arithmetic differential equations of order 4 and 5

2.3. The family of Calabi-Yau quintics 26

2.4. $\mathrm{Sp}_{4}$ modularity 29

2.5. Ramanujan-type formulae for $1 / \pi^{2} \quad 32$

3. Lattice sums and Mahler measures 35

3.1. Dirichlet $L$-series and Mahler measures $\quad 35$

3.2. Quadruple lattice sums 38

Bibliography $\quad 42$

Work is supported by Australian Research Council grant DP110104419.

AMS 2010 Mathematics Subject Classification. Primary 33C20; Secondary 05A19, 11B65, 11F11, 11J82, 11M06, 11Y60, 14H52, 14J32, 33C75, 33F10, 34M50, 40G99, 65B10, 65Q05. 


\section{Introduction}

Arithmetic, known nowadays more usually as number theory, is the heart and one of the oldest parts of mathematics. Carl Friedrich Gauss, arguably the greatest mathematician of all the time, called mathematics the 'Queen of science' and he referred to Number Theory as the 'Queen of mathematics'. Number theory is famous for having many problems which any school student can understand but whose solutions require deep methods of modern mathematics. A famous example is Fermat's Last Theorem about the insolvability of the equation $x^{n}+y^{n}=z^{n}$ in positive integers with $n>2$, which was not actually proved by Fermat himself but quite recently by Andrew Wiles. Another example, which remains an open problem, is the Riemann Hypothesis about the zeros of the function

$$
\zeta(s):=\sum_{n=1}^{\infty} \frac{1}{n^{s}}
$$

It is a well-accepted fact that the central problems of number theory are very hard and as such require methods from many different branches of mathematics; the development of these methods is highly influential on mathematics in general.

Among the important tools for solving problems in number theory are the so-called special functions, whose origin and ultimate importance in mathematical physics was, for a long time, the main reason for their development. Special functions usually appear in exactly solvable problems of physical origin [10], but remarkably they are of definite value in number theory as well. All classical mathematical constants [55] such as $e, \pi$, values of the logarithms, generalized polylogarithmic functions, zeta values $\zeta(s)$ at integers $s>1$, come as special values of certain special functions (not necessarily uniquely chosen).

An important subclass of special functions is the hypergeometric functions [13], [61], [130] defined by the series

$$
{ }_{m} F_{m-1}\left(\begin{array}{cccc}
a_{1}, & a_{2}, & \ldots, & a_{m} \\
& b_{2}, & \ldots, & b_{m}
\end{array} \mid z\right)=\sum_{n=0}^{\infty} \frac{\left(a_{1}\right)_{n}\left(a_{2}\right)_{n} \cdots\left(a_{m}\right)_{n}}{\left(b_{2}\right)_{n} \cdots\left(b_{m}\right)_{n}} \frac{z^{n}}{n !},
$$

in the unit disc $|z|<1$, and analytically continued to the whole $\mathbb{C}$-plane with cut along the ray $[1,+\infty)$. Here

$$
(a)_{n}=\frac{\Gamma(a+n)}{\Gamma(a)}= \begin{cases}a(a+1) \cdots(a+n-1) & \text { if } n \geqslant 1, \\ 1 & \text { if } n=0,\end{cases}
$$

denotes the Pochhammer symbol (or rising factorial). The ${ }_{m} F_{m-1}$ function in (1) satisfies the linear differential equation

$$
\left(\theta \prod_{j=2}^{m}\left(\theta+b_{j}-1\right)-z \prod_{j=1}^{m}\left(\theta+a_{j}\right)\right) y=0, \quad \theta=z \frac{\mathrm{d}}{\mathrm{d} z},
$$

of order $m$, which is an example of Picard-Fuchs differential equation [152]. The theory of hypergeometric functions allows for the unification of many recent achievements in number theory including, for example, Apéry's mysterious proof of the irrationality of $\zeta(3)$ [106] and Ramanujan's rapidly convergent series for $\pi$ [30]. 
It is not surprising to see that number theory in fact requires only a certain subclass of hypergeometric functions, the functions which meet several arithmetic requirements; for example, the parameters of (1) have to be rational or algebraic numbers. It is these functions which we refer to as arithmetic hypergeometric series. The idea to formalise such arithmetic conditions was born on the border of physical String Theory and mathematical Algebraic Geometry. The so-called 'mirror symmetry' produces a natural duality between certain geometric objects; on one side of this duality we have Calabi-Yau manifolds whose periods satisfy certain special Picard-Fuchs differential equations, and the arithmetic data encoded in these equations (as expected) gives one a way to reconstruct the dual side. The joint paper [6] of G. Almkvist and this author is one of the first attempts to put the theory of such Calabi-Yau differential equations on the market of number theory. Further development of this subject along with its linking to other problems in number theory [154], [181], algebraic geometry [4], mathematical physics [73], and other parts of mathematical science, is of great importance in contemporary mathematics.

The modest goal of this survey is to demonstrate how the arithmetic hypergeometric series link certain seemingly unrelated to each other research subjects as well as to explain the underlying arithmetic and analytical techniques. More specifically, we address the following directions:

(1) arithmetic properties of the values of Riemann's zeta function function $\zeta(s)$ at integers $s>1$ and their generalisations;

(2) arithmetic significance of Calabi-Yau differential equations and generalised Ramanujan-type series for $\pi$; and

(3) hypergeometric and special-function evaluations of Mahler measures.

Section 1 discusses the arithmetic properties of the values of Riemann's zeta function. The arithmetic nature of $\zeta(s)$ at integers $s=2,3,4, \ldots$ (zeta values) is well understood for even $s$, thanks to Euler's evaluation of $\zeta(2 k)$ yielding $\zeta(2 k) / \pi^{2 k} \in \mathbb{Q}$ and Lindemann's 1881 proof of the transcendence of $\pi$. For odd $s$, we have results only for irrationality (and not transcendence, even though the numbers are all conjecturally transcendental). After Apéry's unanticipated 1978 proof of the irrationality of $\zeta(3)$, quite recent results of K. Ball and T. Rivoal [15] and of the author [163], [173] show that there are infinitely many irrational numbers in the set $\zeta(3), \zeta(5), \zeta(7), \ldots$ and much more. For example, we now know that each set

$$
\zeta(s+2), \zeta(s+4), \ldots, \zeta(8 s-3), \zeta(8 s-1)
$$

with odd $s>1$ contains at least one irrational number, as well as at least one of the four numbers $\zeta(5), \zeta(7), \zeta(9), \zeta(11)$ is irrational. The hypergeometric techniques used in the proofs allows one to get similar results for the values of other Dirichlet's series [119], for $q$-analogues of zeta values [84], [172] and, more generally, for the values of many other classical $q$-series [37], [39], [97].

It is Section 2 where we give a formal definition of Calabi-Yau differential equations and overview their monodromy properties. The huge database of such equations is now tabulated in [3], and one of the problems in the subject is to understand the relations between different examples of such special equations and their solutions, which are manifestly arithmetic hypergeometric series, by means of algebraic transformations. An example of such transformations extracted from the recent 
work [5] is as follows. If we define two double hypergeometric series,

$$
F(z)=\sum_{n=0}^{\infty} z^{n}\left(\sum_{k=0}^{n}(-1)^{k}\left(\begin{array}{l}
n \\
k
\end{array}\right) \frac{\left(\frac{1}{2}\right)_{k}^{2}}{k !^{2}}\right)^{2}
$$

and

$$
\widehat{F}(z)=\sum_{n=0}^{\infty} z^{n} \frac{\left(\frac{1}{4}\right)_{n}\left(\frac{3}{4}\right)_{n}}{n !^{2}} \sum_{k=0}^{n}(-1)^{k}\left(\begin{array}{l}
n \\
k
\end{array}\right) \frac{\left(\frac{1}{4}\right)_{k}\left(\frac{3}{4}\right)_{k}}{k !^{2}},
$$

then

$$
F(z)=\frac{1}{\sqrt{1-6 z+z^{2}}} \widehat{F}\left(\frac{-16 z(1-z)^{2}}{\left(1-6 z+z^{2}\right)^{2}}\right) .
$$

In Section 2 we also discuss generalised Ramanujan formulae for $\pi$. Proofs of the classical Ramanujan formulae for $1 / \pi$, such as [111]

$$
\begin{aligned}
\sum_{n=0}^{\infty} \frac{\left(\frac{1}{2}\right)_{n}^{3}}{n !^{3}}(6 n+1) \frac{1}{4^{n}} & =\frac{4}{\pi} \\
\sum_{n=0}^{\infty} \frac{\left(\frac{1}{2}\right)_{n}\left(\frac{1}{6}\right)_{n}\left(\frac{5}{6}\right)_{n}}{n !^{3}}(5418 n+263) \frac{(-1)^{n}}{80^{3 n}} & =\frac{640 \sqrt{15}}{3 \pi},
\end{aligned}
$$

are now uniform thanks to the modular and hypergeometric machinery [30], [16], [181]. A similar formula, namely,

$$
\sum_{n=0}^{\infty} \frac{\left(\frac{1}{6}\right)_{n}\left(\frac{1}{2}\right)_{n}\left(\frac{5}{6}\right)_{n}}{n !^{3}}(545140134 n+13591409) \cdot \frac{(-1)^{n}}{53360^{3 n+2}}=\frac{3}{2 \pi \sqrt{10005}}
$$

due to the Chudnovskys [43], was used by Bellard in December 2009 to compute a record 2.7 trillion digits of $\pi$ - on a single workstation. Such series were discovered by Ramanujan in 1914 but the first complete proofs were obtained only in the 1980s [30]. However, extensions of these formulae (with five Pochhammer symbols in the numerator and $n !^{5}$ in the denominator) were only recently discovered by J. Guillera [65]-[68] and by G. Almkvist and J. Guillera [2]. There are eleven known formulae of this kind of which only four are proved rigorously (using hypergeometric algorithms) while seven, such as

$$
\sum_{n=0}^{\infty} \frac{\left(\frac{1}{2}\right)_{n}\left(\frac{1}{3}\right)_{n}\left(\frac{2}{3}\right)_{n}\left(\frac{1}{6}\right)_{n}\left(\frac{5}{6}\right)_{n}}{n !^{5}}\left(5418 n^{2}+693 n+29\right) \frac{(-1)^{n}}{80^{3 n}} \stackrel{?}{=} \frac{128 \sqrt{5}}{\pi^{2}}
$$

and

$$
\sum_{n=0}^{\infty} \frac{(6 n) !}{n !^{6}}\left(532 n^{2}+126 n+9\right) \frac{1}{10^{6 n}} \stackrel{?}{=} \frac{375}{4 \pi^{2}},
$$

remain beyond reach of the current methods. It is important to understand the structure of such formulae and to develop techniques for proving them. The corresponding hypergeometric differential equations are special cases of the Calabi-Yau differential equations. 
Section 3 deals with special Mahler measures. The (logarithmic) Mahler measure of a Laurent polynomial

$$
P\left(x_{1}, \ldots, x_{n}\right) \in \mathbb{C}\left[x_{1}^{ \pm 1}, \ldots, x_{n}^{ \pm 1}\right]
$$

is defined by

$$
\mathrm{m}(P):=\int \cdots \int_{[0,1]^{n}} \log \left|P\left(e^{2 \pi i \theta_{1}}, \ldots, e^{2 \pi i \theta_{n}}\right)\right| \mathrm{d} \theta_{1} \cdots \mathrm{d} \theta_{n} .
$$

The measure first introduced by Hermite 150 years ago and studied carefully by Mahler in the 1960s is crucial to understanding the fine structure of the zeros of integer polynomials and so of transcendence. In 1998 D. W. Boyd - with a very large amount of computation - conjectured [34] a number of striking and unexpected relations between the Dirichlet $L$-series (that is, generalised zeta values) of elliptic curves and the Mahler measures of polynomials with zero varieties that correspond to the same elliptic curves.

The first result in this direction was derived by C. Deninger [47] from the Beilinson conjectures; he showed that, up to a rational multiple $r$,

$$
\mathrm{m}\left(1+x+x^{-1}+y+y^{-1}\right)=r L^{\prime}(E, 0)=r \frac{15}{4 \pi^{2}} L(E, 2),
$$

where $E$ is an elliptic curve of conductor 15. Numerically [34], the multiple seems to be equal to 1 , and by appealing to the modularity theorem for elliptic curves we can write the expected equality as

$$
\begin{aligned}
& \mathrm{m}\left(1+x+x^{-1}+y+y^{-1}\right) \\
& \quad=\frac{540}{\pi^{2}} \sum_{\substack{n_{i}=-\infty \\
i=1,2,3,4}}^{\infty} \frac{(-1)^{n_{1}+n_{2}+n_{3}+n_{4}}}{\left(\left(6 n_{1}+1\right)^{2}+3\left(6 n_{2}+1\right)^{2}+5\left(6 n_{3}+1\right)^{2}+15\left(6 n_{4}+1\right)^{2}\right)^{2}} .
\end{aligned}
$$

$K$-theory serves as a natural machinery for attacking Boyd's conjectures [120], [121], [36], [98], but major progress in this direction is due to combining the approach with modular-function techniques. This was developed by F. Rodríguez-Villegas and later by M. J. Bertin, D. W. Boyd and others [20], [35], [120]. The requirement of modularity is however very restrictive. It turns out that the majority of Boyd's conjectures can be rephrased as identities for hypergeometric series. This approach was used by M. Lalín and M. D. Rogers [87], [123] to derive several new identities for the Mahler measures. For example, the hypergeometric evaluation [123]

$$
\mathrm{m}\left(1+x+x^{-1}+y+y^{-1}\right)=4 \sum_{n=0}^{\infty}\left(\begin{array}{c}
2 n \\
n
\end{array}\right)^{2} \frac{(1 / 16)^{2 n+1}}{2 n+1}
$$

reduces the evaluation (10) to verifying that the quadruple sum is the value of the hypergeometric series on the right-hand side of (11). Introducing hypergeometric techniques is a key to a rigorous proof of (10) in [125] and also to some other longstanding conjectures from Boyd's list [124]. 
The consequence of (10) and (11),

$$
\begin{aligned}
& \frac{540}{\pi^{2}} \sum_{\substack{n_{i}=-\infty \\
i=1,2,3,4}}^{\infty} \frac{(-1)^{n_{1}+n_{2}+n_{3}+n_{4}}}{\left(\left(6 n_{1}+1\right)^{2}+3\left(6 n_{2}+1\right)^{2}+5\left(6 n_{3}+1\right)^{2}+15\left(6 n_{4}+1\right)^{2}\right)^{2}} \\
& =4 \sum_{n=0}^{\infty}\left(\begin{array}{c}
2 n \\
n
\end{array}\right)^{2} \frac{(1 / 16)^{2 n+1}}{2 n+1},
\end{aligned}
$$

is a deep analytic result, because it relates a complicated lattice sum to a simple ${ }_{3} F_{2}$ hypergeometric function. Lattice sums have been extensively studied in physics, where they often arise when calculating electrostatic potentials of crystal lattices (for instance, see [30], [59], [63] and [159]). It cannot be coincidental that the evaluation (12) bears a striking resemblance to a famous formula for Catalan's constant discovered by Ramanujan [55]:

$$
\frac{1}{\pi} \sum_{n=0}^{\infty} \frac{(-1)^{n}}{(2 n+1)^{2}}=\sum_{n=0}^{\infty}\left(\begin{array}{c}
2 n \\
n
\end{array}\right)^{2} \frac{(1 / 4)^{2 n+1}}{2 n+1}
$$

Catalan's constant is one of the simplest arithmetic quantities whose irrationality is still unproven. This fact that the right-hand sides of (12) and (13) are arithmetic values of the same hypergeometric function makes us confident in claiming that many Mahler measure identities can be proved by the arithmetic hypergeometric techniques. It serves perfectly to illustrate the need for a better understanding of such sometimes rigorous and sometimes experimental evaluations.

For many years, Anatolii Alekseevich Karatsuba was expressing his interest in my arithmetic-hypergeometric research. This was much more than a moral support, because in 2006-2008 I worked under his leadership in the Division of Number Theory at the Steklov Institute of Mathematics. His sudden death is a big loss for number theory and mathematics, but it is at the same time my personal loss of a teacher and friend. This survey is my tribute to Anatolii Alekseevich.

My personal enjoyment of arithmetic hypergeometric series and my knowledge on the subject would be not possible without my colleagues, collaborators and friends. I use this opportunity to express my gratitude to G. Almkvist, A. Aptekarev, D. Bertrand, J. Borwein, P. Bundschuh, H.H. Chan, V. Golyshev, S. Fischler, J. Guillera, M. Huttner, C. Krattenthaler, L. Long, T. Matalo-aho, A. Mellit, Yu. Nesterenko, Y. Ohno, G. Rhin, T. Rivoal, I. Rochev, M. Rogers, V. Sorokin, V. Spiridonov, A. Straub, K. Väänänen, W. Van Assche, D. van Straten, C. Viola, M. Waldschmidt, J. Wan, O. Warnaar, Y. Yang, D. Zagier, and D. Zeilberger.

\section{Arithmetic of the values of Riemann's zeta function}

1.1. Apéry's theorem. Investigation of sums of the form

$$
\zeta(s)=\sum_{n=1}^{\infty} \frac{1}{n^{s}}
$$

for positive integers $s$ goes back to L. Euler [53], [54]. In particular, he proved the divergence of the series in (14) when $s=1$ and its convergence when $s>1$, as well 
as his famous formulae

$$
2 \sum_{n=1}^{\infty} \frac{1}{n^{2 k}}=-\frac{(2 \pi i)^{2 k} B_{2 k}}{(2 k) !} \quad \text { for } \quad k=1,2,3, \ldots
$$

The latter relate the values of the series at positive even $s$ to the Archimedean constant $\pi=3.14159265 \ldots$ (see $[55 ; \S 1.4]$ ) and the Bernoulli numbers $B_{s} \in \mathbb{Q}$, which can be defined by means of the generating function

$$
\frac{z}{e^{z}-1}=1-\frac{z}{2}+\sum_{s=2}^{\infty} B_{s} \frac{z^{s}}{s !}=1-\frac{z}{2}+\sum_{k=1}^{\infty} B_{2 k} \frac{z^{2 k}}{(2 k) !} .
$$

In 1882 F. Lindemann [90] established the transcendence of $\pi$, thus, the transcendence of $\zeta(s)$ for $s$ even.

It was only one century after Euler, when B. Riemann [115] considered the series in (14) as a function of complex variable $s$. In the domain $\operatorname{Re} s>1$, the series represents an analytical function which can be continued to the whole complex plane to the meromorphic function $\zeta(s)$. It is this analytical continuation, as well as some other important properties of the function $\zeta(s)$, which were discovered by Riemann in his memoir on prime numbers. Riemann's zeta function and its generalisations play a fundamental role in analytic number theory [145]. In what follows we only discuss arithmetic and analytical properties of the Euler sums $\zeta(s)$ in (14) for positive integers $s>1$, and their generalisations. For brevity, we will call the quantities (14) zeta values, and also even and odd zeta values depending on the parity of positive integer $s$.

As noted above, the transcendence (hence the irrationality) of the even zeta values follow from the classical results of Euler and Lindemann. Similar to (15) formulae for odd zeta values are not known and, presumably, the number $\zeta(2 k+$ $1) / \pi^{2 k+1}$ is not rational for any integer $k \geqslant 1$. The arithmetic nature of odd zeta values seemed to be impregnable till 1978 when R. Apéry produced a sequence of rational approximations which showed the irrationality of $\zeta(3)$.

History of this discovery as well as a rigorous mathematical justification of Apéry's claims are exposed in [106]. Number $\zeta(3)$ is known nowadays as the Apéry constant (see, for example, $[55 ; \S 1.6]$ ). The rational approximations to $\zeta(3)$ given by Apéry have the form $v_{n} / u_{n} \in \mathbb{Q}$ for $n=0,1,2, \ldots$, where the denominators $\left\{u_{n}\right\}=\left\{u_{n}\right\}_{n=0,1, \ldots}$ as well as the numerators $\left\{v_{n}\right\}=\left\{v_{n}\right\}_{n=0,1, \ldots}$ satisfy the same polynomial recursion

$$
(n+1)^{3} u_{n+1}-(2 n+1)\left(17 n^{2}+17 n+5\right) u_{n}+n^{3} u_{n-1}=0
$$

with the initial data

$$
u_{0}=1, \quad u_{1}=5, \quad \text { and } \quad v_{0}=0, \quad v_{1}=6 .
$$

Then

$$
\lim _{n \rightarrow \infty} \frac{v_{n}}{u_{n}}=\zeta(3)
$$


nonetheless an important but unexpected (from the recursion (16) point of view) circumstance is the inclusions

$$
u_{n}=\sum_{k=0}^{n}\left(\begin{array}{l}
n \\
k
\end{array}\right)^{2}\left(\begin{array}{c}
n+k \\
k
\end{array}\right)^{2} \in \mathbb{Z}, \quad D_{n}^{3} v_{n} \in \mathbb{Z}, \quad n=0,1,2, \ldots,
$$

where $D_{n}$ denotes the least common multiple of $1,2, \ldots, n$ (and $D_{0}=1$ for completeness). Application of Poincaré's theorem (see, for example, [62]) to the difference equation (16) leads one to the limiting relations

$$
\begin{gathered}
\lim _{n \rightarrow \infty}\left|u_{n} \zeta(3)-v_{n}\right|^{1 / n}=(\sqrt{2}-1)^{4}, \\
\lim _{n \rightarrow \infty}\left|u_{n}\right|^{1 / n}=\lim _{n \rightarrow \infty}\left|v_{n}\right|^{1 / n}=(\sqrt{2}+1)^{4}
\end{gathered}
$$

in accordance with $(18)$; here numbers $(\sqrt{2}-1)^{4}$ and $(\sqrt{2}+1)^{4}$ are roots of the characteristic polynomial $\lambda^{2}-34 \lambda+1$ of the recursion (16). The information gathered about the properties of the sequences $\left\{u_{n}\right\}$ and $\left\{v_{n}\right\}$ demonstrates that number $\zeta(3)$ cannot be rational. Indeed, under assumption that $\zeta(3)=a / b$ for some $a, b \in \mathbb{Z}$, the linear forms $r_{n}=b D_{n}^{3}\left(u_{n} \zeta(3)-v_{n}\right)$ are integers which are nonzero by (20). On the other hand, $D_{n}^{1 / n} \rightarrow e$ as $n \rightarrow \infty$ in accordance with the prime number theorem (see, for example, [145; Chap. II, §3]); therefore,

$$
\lim _{n \rightarrow \infty}\left|r_{n}\right|^{1 / n}=e^{3}(\sqrt{2}-1)^{4}=0.59126300 \ldots<1,
$$

and for all $n$ sufficiently large this estimate contradicts the bound $\left|r_{n}\right| \geqslant 1$ valid for nonzero integers $r_{n}$. Furthermore, the additional limiting relations (21) and a standard argument (see, for example, [75; Lemma 3.1]) allow one to measure the irrationality of the Apéry constant quantitatively:

$$
\mu(\zeta(3)) \leqslant 1+\frac{4 \log (\sqrt{2}+1)+3}{4 \log (\sqrt{2}+1)-3}=13.41782023 \ldots .
$$

Here and below, the irrationality exponent $\mu(\alpha)$ of a real irrational number $\alpha$ is the quantity

$$
\begin{gathered}
\mu=\mu(\alpha)=\inf \left\{c \in \mathbb{R}: \text { the inequality }|\alpha-a / b| \leqslant|b|^{-c}\right. \text { has } \\
\text { finitely many solutions in } a, b \in \mathbb{Z}\}
\end{gathered}
$$

when $\mu(\alpha)<+\infty$, we say that $\alpha$ is a non-Liouvillian number.

Apéry original derivation (namely, relations (16)-(21)) were so mysterious that the interest to Apéry's theorem remains strong till present time. The phenomenon of the sequence of Apéry's rational approximations was reconsidered time and again from points of view of different methods (see [21], [24], [57], [72], [75], [101], [108], [113], [132], [134], [135], [144], [154], [155], [160], and [182]). The new approaches led to strengthening Apéry's result quantitatively, new estimates for the irrationality exponent of $\zeta(3)$ were deduced (the last stages of a competition in this direction are the works [76] and [113]). We now indicate explicit formulae for the sequence 
$u_{n} \zeta(3)-v_{n}$ that play an important role in our further discussion: Beukers' representation $[21]$

$$
u_{n} \zeta(3)-v_{n}=\iiint_{[0,1]^{3}} \frac{x^{n}(1-x)^{n} y^{n}(1-y)^{n} z^{n}(1-z)^{n}}{(1-(1-x y) z)^{n+1}} \mathrm{~d} x \mathrm{~d} y \mathrm{~d} z
$$

in the form of multiple real integral, as well as the Gutnik-Nesterenko series [72], [101]

$$
u_{n} \zeta(3)-v_{n}=-\left.\frac{1}{2} \sum_{\nu=1}^{\infty} \frac{\mathrm{d}}{\mathrm{d} t}\left(\frac{(t-1)(t-2) \cdots(t-n)}{t(t+1)(t+2) \cdots(t+n)}\right)^{2}\right|_{t=\nu}
$$

and the Ball series [15]

$$
u_{n} \zeta(3)-v_{n}=\left.n !^{2} \sum_{\nu=1}^{\infty}\left(t+\frac{n}{2}\right) \frac{(t-1) \cdots(t-n) \cdot(t+n+1) \cdots(t+2 n)}{t^{4}(t+1)^{4} \cdots(t+n)^{4}}\right|_{t=\nu} .
$$

We remark that on invoking his 'acceleration convergence' method, Apéry [12], [106] also established the irrationality of $\zeta(2)$ without use of the formula $\zeta(2)=$ $\pi^{2} / 6$. This time the denominators $\left\{u_{n}^{\prime}\right\}$ and numerators $\left\{v_{n}^{\prime}\right\}$ of his linear approximation forms $u_{n}^{\prime} \zeta(2)-v_{n}^{\prime}, n=0,1,2, \ldots$, satisfy the recursion

$$
(n+1)^{2} u_{n+1}-\left(11 n^{2}+11 n+3\right) u_{n}-n^{2} u_{n-1}=0
$$

with the initial data

$$
u_{0}^{\prime}=1, \quad u_{1}^{\prime}=3, \quad v_{0}^{\prime}=0, \quad v_{1}^{\prime}=5 .
$$

Then

$$
u_{n}^{\prime}=\sum_{k=0}^{n}\left(\begin{array}{l}
n \\
k
\end{array}\right)^{2}\left(\begin{array}{c}
n+k \\
k
\end{array}\right) \in \mathbb{Z}, \quad D_{n}^{2} v_{n}^{\prime} \in \mathbb{Z}, \quad n=0,1,2, \ldots
$$

and

$$
\begin{aligned}
& \lim _{n \rightarrow \infty}\left|u_{n}^{\prime} \zeta(2)-v_{n}^{\prime}\right|^{1 / n}=\left(\frac{\sqrt{5}-1}{2}\right)^{5}<e^{-2}, \\
& \lim _{n \rightarrow \infty}\left|u_{n}\right|^{1 / n}=\lim _{n \rightarrow \infty}\left|v_{n}\right|^{1 / n}=\left(\frac{\sqrt{5}+1}{2}\right)^{5} .
\end{aligned}
$$

This sequence of approximations results in the estimate

$$
\mu(\zeta(2))=\mu\left(\pi^{2}\right) \leqslant 1+\frac{5 \log ((\sqrt{5}+1) / 2)+2}{5 \log ((\sqrt{5}+1) / 2)-2}=11.85078219 \ldots
$$

for the irrationality exponent of $\pi^{2}$. Apéry's approximations to $\zeta(2)$ can be given by the double real integral [21]

$$
u_{n}^{\prime} \zeta(2)-v_{n}^{\prime}=(-1)^{n} \iint_{[0,1]^{2}} \frac{x^{n}(1-x)^{n} y^{n}(1-y)^{n}}{(1-x y)^{n+1}} \mathrm{~d} x \mathrm{~d} y,
$$


as well as by the hypergeometric series

$$
u_{n}^{\prime} \zeta(2)-v_{n}^{\prime}=\left.(-1)^{n} \sum_{\nu=1}^{\infty} \frac{n ! \cdot(t-1)(t-2) \cdots(t-n)}{t^{2}(t+1)^{2}(t+2)^{2} \cdots(t+n)^{2}}\right|_{t=\nu}
$$

Apéry theorem is in essence the very first step in approaching the following problem (which can be undoubtedly called folklore; see, for example, [129; Concluding remarks] for a record): prove that numbers $\zeta(2 k+1)$ are irrational for $k=1,2,3, \ldots$.

Unfortunately, natural generalisations of Apéry's construction lead one to linear forms involving values of the zeta function at both odd and even points. The latter circumstance prevented to obtain results about the irrationality of $\zeta(s)$ for odd $s \geqslant 5$. It was only in 2000, when T. Rivoal [116] used a general Ball's representation (24) to construct linear forms involving only the odd zeta values; the construction allowed him to prove the following result.

Theorem 1. Of the numbers

$$
\zeta(3), \zeta(5), \zeta(7), \zeta(9), \zeta(11), \ldots
$$

infinitely many are irrational. More precisely, the dimension $\delta(s)$ of the spaces which are generated by $1, \zeta(3), \zeta(5), \ldots, \zeta(s-2), \zeta(s)$ over $\mathbb{Q}$, for $s$ odd, satisfies the estimate

$$
\delta(s) \geqslant \frac{\log s}{1+\log 2}(1+o(1)) \quad \text { as } s \rightarrow \infty .
$$

Rivoal's linear approximation forms in [116] are given by

$$
\begin{gathered}
F_{n}=F_{s, r, n}=\left.n !^{s+1-2 r} \sum_{\nu=1}^{\infty}\left(t+\frac{n}{2}\right) \frac{\prod_{j=1}^{r n}(t-j) \cdot \prod_{j=1}^{r n}(t+n+j)}{\prod_{j=0}^{n}(t+j)^{s+1}}\right|_{t=\nu}, \\
s \text { is odd, }
\end{gathered}
$$

where the auxiliary parameter $r<s / 2$ is of order $r \sim s / \log ^{2} s$; in particular, the series $F_{3,1, n}$ coincides with representation (24) of Apéry's sequence. Decomposing the summand, which is a rational function of parameter $t$, into the sum of partial fractions and using the ideas from [101] and [103] one can show the arithmetic inclusions

$$
2 D_{n}^{s+1} F_{n} \in \mathbb{Z} \zeta(s)+\mathbb{Z} \zeta(s-2)+\cdots+\mathbb{Z} \zeta(5)+\mathbb{Z} \zeta(3)+\mathbb{Z}
$$

Furthermore, the explicit formulae (32) for the linear forms in odd zeta values allow one to compute the asymptotic behaviour of the forms and their coefficients as $n \rightarrow \infty$. The final step in Rivoal's proof is application of Nesterenko's linear independence criterion [100].

The fact that the quantities in $(32)$ are $\mathbb{Q}$-linear forms in 1 and zeta values of the same parity, is related to a special symmetry of the rational summand in (32). Possible applications of less exotic rational functions are discussed in the works 
[72], [77], and [117]: the results there are about dimensions of the spaces generated over $\mathbb{Q}$ by the polylogarithmic values

$$
\operatorname{Li}_{s}(z)=\sum_{n=1}^{\infty} \frac{z^{n}}{n^{s}}
$$

at a rational point $z, 0<|z| \leqslant 1$.

In spite of the fact that the proof of Rivoal's theorem is indeed a certain generalization of the construction from the proofs of Apéry's theorem, Rivoal's result provides only a partial solution to the problem of the irrationality of odd zeta values. For the zeta value $\zeta(s)$ which is next irrational after $\zeta(3)$, Rivoal's theorem [15] only produces the range $5 \leqslant s \leqslant 169$. Differentiation of rational summand (like in representation (23)) allows one to construct $\mathbb{Q}$-linear forms in odd zeta values which do not involve $\zeta(3)$. This leads one [163], [118] to the result that at least one of the nine odd zeta values $\zeta(5), \zeta(7), \ldots, \zeta(21)$ is irrational. Finally, on invoking the most general form of the construction proposed in Rivoal's works as well as the arithmetic method (discussed, for example, in [42], [126], and [75]), which is traditionally used for sharpening bounds of irrationality measures, we prove in [162], [173] the following result.

Theorem 2. One of the numbers

$$
\zeta(5), \zeta(7), \zeta(9), \text { and } \zeta(11)
$$

is irrational.

We notice that the techniques used in the proof is also successfull in other arithmetic problems: the paper [119] establishes analogues of Theorems 1 and 2 for the values of Dirichlet's beta function

$$
\beta(s)=\sum_{n=0}^{\infty} \frac{(-1)^{n}}{(2 n+1)^{s}}
$$

at even $s \geqslant 2$. In the joint paper [58], we give a certain strengthening (and a new proof) of Nesterenko's linear independence criterion from [100]; applying the result we sharpen the ranges of the argument of the zeta function from [15] and [163].

1.2. Hypergeometric series and multiple integrals. Beukers' proof [21] of the irrationality of both $\zeta(2)$ and $\zeta(3)$, which makes use of the integral representations (30) and (22), is simple and short. This served as main grounds for further applications of multiple integrals in the quantitative improvements and generalisations of Apéry's results (see [50], [75], [76], [112], [113], [140], [141], [142], and [144]). O. Vasilenko in [140] proposed to consider the following family of $s$-fold multiple integrals which generalise the Beukers integrals:

$$
J_{s, n}=\int_{[0,1]^{s}} \ldots \int \frac{\prod_{j=1}^{s} x_{j}^{n}\left(1-x_{j}\right)^{n}}{Q_{s}\left(x_{1}, \ldots, x_{s}\right)^{n+1}} \mathrm{~d} x_{1} \cdots \mathrm{d} x_{s},
$$

where

$$
Q_{s}\left(x_{1}, \ldots, x_{s}\right)=1-x_{1}\left(1-x_{2}\left(1-\cdots\left(1-x_{s-1}\left(1-x_{s}\right)\right) \cdots\right)\right)
$$


The first progress in this direction was the paper [142] of D. Vasil'ev who studied the integrals $J_{4, n}, J_{5, n}$ and proved that

$$
4 D_{n}^{4} J_{4, n} \in \mathbb{Z} \zeta(4)+\mathbb{Z} \zeta(2)+\mathbb{Z}, \quad D_{n}^{5} J_{5, n} \in \mathbb{Z} \zeta(5)+\mathbb{Z} \zeta(3)+\mathbb{Z},
$$

as well as that the linear forms in (33) tend (reasonably fast) to zero as $n \rightarrow \infty$ (unfortunately, not sufficiently fast to conclude on the new irrationality of zeta values). The inclusions $D_{n}^{2} J_{2, n} \in \mathbb{Z} \zeta(2)+\mathbb{Z}$ and $D_{n}^{3} J_{3, n} \in \mathbb{Z} \zeta(3)+\mathbb{Z}$ established by Beukers in [21], and (35) gave Vasil'ev grounds to conjecture that

$$
\begin{gathered}
2^{s-2} D_{n}^{s} J_{s, n} \in \mathbb{Z} \zeta(s)+\mathbb{Z} \zeta(s-2)+\cdots+\mathbb{Z} \zeta(4)+\mathbb{Z} \zeta(2)+\mathbb{Z} \quad \text { for } s \text { even }, \\
D_{n}^{s} J_{s, n} \in \mathbb{Z} \zeta(s)+\mathbb{Z} \zeta(s-2)+\cdots+\mathbb{Z} \zeta(5)+\mathbb{Z} \zeta(3)+\mathbb{Z} \quad \text { for } s \text { odd. }
\end{gathered}
$$

In spite of validity of this expectation for $s=2,3,4,5$, the confidence of the author of [142] in the truth of (36) for all $s$ was not shared by everybody. The reason for that was another wrong conjecture, namely, $2^{s-2} D_{n}^{s} J_{s, n} \in \mathbb{Z} \zeta(s)+\mathbb{Z}$ for $s$ even and $D_{n}^{s} J_{s, n} \in \mathbb{Z} \zeta(s)+\mathbb{Z}$ for $s$ odd, proposed by Vasil'ev in his previous work [141]. One of the first steps in answering Vasil'ev's question in the affirmative was the following partial (up to an extra multiple $2 D_{n}$ ) result [166], [171], [173].

Theorem 3. For every integer $s \geqslant 2$ and $n=0,1,2, \ldots$, the identity

$$
J_{s, n}=F_{s, n}
$$

is true, where

$$
F_{s, n}=\left.n !^{s-1} \sum_{\nu=1}^{\infty}(-1)^{(s+1)(t+n+1)}\left(t+\frac{n}{2}\right) \frac{\prod_{j=1}^{n}(t-j) \cdot \prod_{j=1}^{n}(t+n+j)}{\prod_{j=0}^{n}(t+j)^{s+1}}\right|_{t=\nu} .
$$

In particular, the following inclusions take place:

$$
\begin{gathered}
2^{s-1} D_{n}^{s+1} J_{s, n} \in \mathbb{Z} \zeta(s)+\mathbb{Z} \zeta(s-2)+\cdots+\mathbb{Z} \zeta(4)+\mathbb{Z} \zeta(2)+\mathbb{Z} \quad \text { for s even }, \\
2 D_{n}^{s+1} J_{s, n} \in \mathbb{Z} \zeta(s)+\mathbb{Z} \zeta(s-2)+\cdots+\mathbb{Z} \zeta(5)+\mathbb{Z} \zeta(3)+\mathbb{Z} \quad \text { for } s \text { odd. }
\end{gathered}
$$

Note that the series (38) is exactly the same as the series (32) for $s$ odd and $r=1$; therefore, identity (37) means the coincidence of the integral construction of $\mathbb{Q}$-linear forms in zeta values with the construction from [116].

Ball's (24) and Rivoal's (32) series are well known in the theory of hypergeometric functions [10], [13], [130]. Formally, a hypergeometric function is defined by the series (1); the condition

$$
\operatorname{Re}\left(a_{1}+a_{2}+\cdots+a_{m}\right)<\operatorname{Re}\left(b_{2}+\cdots+b_{m}\right)
$$

ensures convergence of $(1)$ in the domain $|z| \leqslant 1$ (see, for example, $[13 ; \S 2.1]$ ). An important role in analysis of hypergeometric series is played by summation and transformation formulae. We give as examples the Pfaff-Saalschütz summation theorem

$$
{ }_{3} F_{2}\left(\begin{array}{c|c}
-n, a, b \\
c, 1+a+b-c-n
\end{array} \mid 1\right)=\frac{(c-a)_{n}(c-b)_{n}}{(c)_{n}(c-a-b)_{n}}
$$


(here $n$ is a non-negative integer; see, for example, [130; p. 49, equation (2.3.1.3)]), the limiting case of Dougall's theorem

$$
\begin{aligned}
& { }_{5} F_{4}\left(\begin{array}{ccc|c}
a, 1+\frac{1}{2} a, & b, & c, & d \\
\frac{1}{2} a, & 1+a-b, & 1+a-c, & 1+a-d
\end{array}\right) \\
& =\frac{\Gamma(1+a-b) \Gamma(1+a-c) \Gamma(1+a-d) \Gamma(1+a-b-c-d)}{\Gamma(1+a) \Gamma(1+a-b-c) \Gamma(1+a-b-d) \Gamma(1+a-c-d)}
\end{aligned}
$$

(see $[13 ; \S 4.4])$, and Whipple's transformation

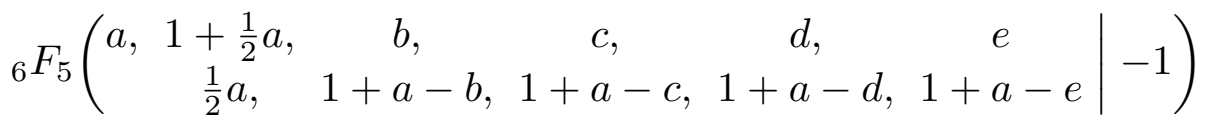

$$
\begin{aligned}
& =\frac{\Gamma(1+a-d) \Gamma(1+a-e)}{\Gamma(1+a) \Gamma(1+a-d-e)} \cdot{ }_{3} F_{2}\left(\begin{array}{c|c}
1+a-b-c, d, e \\
1+a-b, 1+a-c
\end{array}\right)
\end{aligned}
$$

(see $[147]$ and $[13 ; \S 4.4]$ ). Furthermore, the hypergeometric functions possess many integral representations [13], [130]; we mention here the classical Euler-Pochhammer integral for the Gaussian function $(m=1)$

$$
{ }_{2} F_{1}\left(\begin{array}{c|c}
a, b & z
\end{array}\right)=\frac{\Gamma(c)}{\Gamma(b) \Gamma(c-b)} \int_{0}^{1} t^{b-1}(1-t)^{c-b-1}(1-z t)^{-a} \mathrm{~d} t
$$

when $\operatorname{Re} c>\operatorname{Re} b>0$ (see, for example, [130; p. 20, equation (1.6.6)]). Formula (44) is valid for $|z|<1$ and also for any $z \in \mathbb{C}$ whenever $a$ is a positive integer.

In his work [148], F. Whipple called hypergeometric series well-poised if their parameters satisfy the condition

$$
a_{1}+1=a_{2}+b_{2}=\cdots=a_{m}+b_{m} ;
$$

known transformations (like (42) and (43)) usually refer to such series. A special subclass of well-poised hypergeometric series is very-well-poised series, which are subject to the additional condition

$$
a_{2}=\frac{1}{2} a_{1}+1, \quad b_{2}=\frac{1}{2} a_{1} .
$$

A survey on history and applications of (very-) well-poised hypergeometric series is given in [9]. The series (38) (as well as (32)) are very-well-poised:

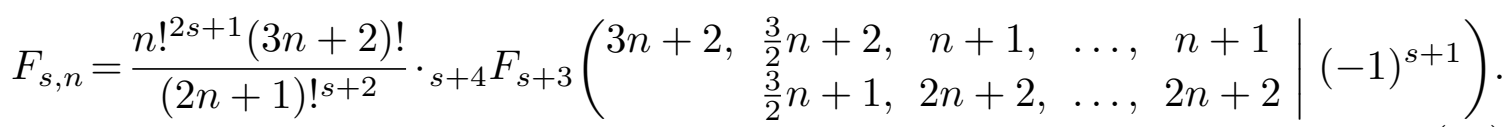

Theorem 3 is a consequence of a more general result [166], [171] about representation of a very-well-poised series as a multiple integral.

Vasil'ev's conjecture (36) was fully solved in the work [79] with the help of Theorem 3. The methods of [79] are based on representation of the sums (45) in the form of multiple hypergeometric series and heavily exploit ideas of the works [142] and [171]; however the technical realisation of the ideas required the authors of [79] large computational work. The series (45) possess different multiple-integral 
representations as well, in particular of Sorokin type (the works [133] and [134] contain number-theoretical applications of such integrals); respective translation theorems for multiple integrals are established by S. Zlobin [156], [157]. At present, there are several works where decomposition of special multiple integrals into linear forms of zeta values and polylogarithms is addressed. This subject already deserves a separate review; we restrict ourselves here by the references [158] and [174], which deal with new generalisations of Beukers' integrals (22) and (30).

It is worth mentioning here that the hypergeometric techniques of the work [79], namely, Andrews' general transformation [8] for terminating very-well-poised series, allowed K. Krattenthaler and T. Rivoal [80] to give a new proof of the theorem from [166], [171] (in particular, of Theorem 3). It is surprising that the transformation, initially designed for $q$-basic hypergeometric series in connection with applications to the theory of partitions - generalisations of the famous Rogers-Ramanujan identities, has found its second birth in arithmetic problems of zeta values. Besides [79], [80], we should mention the works [81] and [175], as well as solution to a problem of A. Schmidt discussed in more details below.

Schmidt observed in [127] that there is a remarkable property of the sequence of Apéry's numbers $\left\{u_{n}\right\}_{n=0,1, \ldots}$ from (19). Namely, if one defines the numbers $\left\{c_{k}\right\}_{k=0,1, \ldots}$ successively from the equalities

$$
u_{n}=\sum_{k=0}^{n}\left(\begin{array}{l}
n \\
k
\end{array}\right)\left(\begin{array}{c}
n+k \\
k
\end{array}\right) c_{k}, \quad n=0,1,2, \ldots,
$$

then these numbers are integral. (The explicit formulae

$$
c_{n}=\left(\begin{array}{c}
2 n \\
n
\end{array}\right)^{-1} \sum_{k=0}^{n}(-1)^{n-k} \frac{2 k+1}{n+k+1}\left(\begin{array}{c}
2 n \\
n-k
\end{array}\right) u_{k}, \quad n=0,1,2, \ldots,
$$

show that expected inclusions are $D_{n} c_{n} \in \mathbb{Z}$.) Later Schmidt himself [128] and, independently, V. Strehl [136] derived the following explicit relation:

$$
c_{n}=\sum_{j=0}^{n}\left(\begin{array}{l}
n \\
j
\end{array}\right)^{3}=\sum_{j}\left(\begin{array}{l}
n \\
j
\end{array}\right)^{2}\left(\begin{array}{c}
2 j \\
n
\end{array}\right), \quad n=0,1,2, \ldots,
$$

experimentally predicted by W. Deuber, W. Thumser, and B. Voigt. In fact, Strehl used in [136] the corresponding identity

$$
\sum_{k=0}^{n}\left(\begin{array}{l}
n \\
k
\end{array}\right)^{2}\left(\begin{array}{c}
n+k \\
k
\end{array}\right)^{2}=\sum_{k=0}^{n}\left(\begin{array}{l}
n \\
k
\end{array}\right)\left(\begin{array}{c}
n+k \\
k
\end{array}\right) \sum_{j=0}^{k}\left(\begin{array}{l}
k \\
j
\end{array}\right)^{3}
$$

as a model for demonstrating various proof techniques for binomial identities. A surprising fact about the sequence (46) is that it was studied already at the end of the 19th century by J. Franel [60], who showed that it satisfies the polynomial recursion

$$
(n+1)^{2} c_{n+1}-\left(7 n^{2}+7 n+2\right) c_{n}-8 n^{2} c_{n-1}=0 .
$$

Schmidt has noticed in [127] that it is likely that the integrality phenomenon related to Apéry's and Franel's numbers take place in a more general situation. This expectation was proven in full generality in [176], [177]. 
Theorem 4. For each integer $r \geqslant 2$, the numerical sequence $\left\{c_{k}^{(r)}\right\}_{k=0,1, \ldots}$ independent of parameter $n$ is defined by the equality

$$
\sum_{k=0}^{n}\left(\begin{array}{l}
n \\
k
\end{array}\right)^{r}\left(\begin{array}{c}
n+k \\
k
\end{array}\right)^{r}=\sum_{k=0}^{n}\left(\begin{array}{l}
n \\
k
\end{array}\right)\left(\begin{array}{c}
n+k \\
k
\end{array}\right) c_{k}^{(r)}, \quad n=0,1,2, \ldots
$$

Then all numbers $c_{k}^{(r)}$ are integral.

But for this case Strehl had only one proof based Using Zeilberger's algorithm of creative telescoping, Strehl proved in [136] the integrality of $c_{k}^{(r)}$ when $r=3$. Schmidt's problem was later stated in the book [64] (Exercise 114 on p. 256) with an indication that H. Wilf had shown the desired integrality of $c_{n}^{(r)}$ for any $r$ but only for any $n \leqslant 9$. The complete proof of Theorem 4 uses a hypergeometric reformulation from [136] of the problem, as well as Andrews' transformation of terminating very-well-poised series [8] mentioned above.

1.3. Simultaneous approximations to $\zeta(2)$ and $\zeta(3)$. In this section we present three hypergeometric constructions of simultaneous rational approximations to $\zeta(2)$ and $\zeta(3)$. This is to not only demonstrate the hypergeometric series in action, but also to show how so seemingly unrelated series give rise to the same numerical approximations. A similar phenomenon, the coincidence of the Gutnik-Nesterenko series (23) and Ball's series (24), was already mentioned in $\S 1.1$. The constructions below depend on an increasing integer parameter $n$.

First [167] we take the rational functions

$$
R_{n}(t)=-\frac{n !^{2} \prod_{j=1}^{n}(t-j)}{\prod_{j=0}^{n}(t+j)^{3}}, \quad R_{n}^{\prime}(t)=\frac{n !^{2} \prod_{j=0}^{n}(t-j)}{\prod_{j=0}^{n}(t+j)^{3}},
$$

and consider the corresponding hypergeometric series

$$
\begin{aligned}
& r_{n}=\left.\sum_{k=1}^{\infty} R_{n}(t)\right|_{t=\nu}=q_{n} \zeta(3)+p_{n} \zeta(2)-s_{n}, \\
& r_{n}^{\prime}=\left.\sum_{k=1}^{\infty} R_{n}^{\prime}(t)\right|_{t=\nu}=q_{n}^{\prime} \zeta(3)+p_{n}^{\prime} \zeta(2)-s_{n}^{\prime},
\end{aligned}
$$

where

$$
q_{n}, q_{n}^{\prime} \in \mathbb{Z}, \quad D_{n} p_{n}, D_{n} p_{n}^{\prime} \in \mathbb{Z}, \quad D_{n}^{3} s_{n}, D_{n}^{3} s_{n}^{\prime} \in \mathbb{Z} .
$$

The standard eliminating argument leads us to the linear forms

$$
\begin{aligned}
q_{n} r_{n}^{\prime}-q_{n}^{\prime} r_{n} & =\left(q_{n} p_{n}^{\prime}-q_{n}^{\prime} p_{n}\right) \zeta(2)-\left(q_{n} s_{n}^{\prime}-q_{n}^{\prime} s_{n}\right)=u_{n} \zeta(2)-v_{n}, \\
p_{n}^{\prime} r_{n}-p_{n} r_{n}^{\prime} & =\left(q_{n} p_{n}^{\prime}-q_{n}^{\prime} p_{n}\right) \zeta(3)-\left(p_{n}^{\prime} s_{n}-p_{n} s_{n}^{\prime}\right)=u_{n} \zeta(3)-w_{n},
\end{aligned}
$$

where, by (48),

$$
D_{n} u_{n} \in \mathbb{Z}, \quad D_{n}^{3} v_{n} \in \mathbb{Z}, \quad D_{n}^{4} w_{n} \in \mathbb{Z} .
$$

The second construction [180] is based on the rational function

$$
\widetilde{R}_{n}(t)=\frac{((t-1)(t-2) \cdots(t-n))^{3}}{n !^{2} \cdot t(t+1) \cdots(t+n)} .
$$


Then hypergeometric approximations to the first three polylogarithms are given by the series

$$
\begin{aligned}
& \widetilde{r}_{n}(z)=\left.\sum_{\nu=1}^{\infty} z^{\nu} \widetilde{R}_{n}(t)\right|_{t=\nu}=\widetilde{u}_{n}(z) \operatorname{Li}_{1}(z)-\widetilde{s}_{n}(z), \\
& \widetilde{r}_{n}^{\prime}(z)=-\left.\sum_{\nu=1}^{\infty} z^{\nu} \frac{\mathrm{d} \widetilde{R}_{n}(t)}{\mathrm{d} t}\right|_{t=\nu}=\widetilde{u}_{n}(z) \operatorname{Li}_{2}(z)-\widetilde{v}_{n}(z), \\
& \widetilde{r}_{n}^{\prime \prime}(z)=\left.\frac{1}{2} \sum_{\nu=1}^{\infty} z^{\nu} \frac{\mathrm{d}^{2} \widetilde{R}_{n}(t)}{\mathrm{d} t^{2}}\right|_{t=\nu}=\widetilde{u}_{n}(z) \operatorname{Li}_{3}(z)-\widetilde{w}_{n}(z),
\end{aligned}
$$

where

$$
\widetilde{u}_{n}(z)=(-1)^{n} \sum_{k=0}^{n}\left(\begin{array}{l}
n \\
k
\end{array}\right)\left(\begin{array}{c}
n+k \\
k
\end{array}\right)^{3}\left(-\frac{1}{z}\right)^{k}
$$

and

$$
\begin{gathered}
z_{1}^{n} \widetilde{u}_{n}(z) \in \mathbb{Z}, \quad\left(z_{1} z_{2}\right)^{n} D_{n} \widetilde{s}_{n}(z) \in \mathbb{Z}, \\
\left(z_{1} z_{2}\right)^{n} D_{n} D_{2 n} \widetilde{v}_{n}(z) \in \mathbb{Z}, \quad\left(z_{1} z_{2}\right)^{n} D_{n} D_{2 n}^{2} \widetilde{w}_{n}(z) \in \mathbb{Z},
\end{gathered}
$$

$z_{1}$ and $z_{2}$ denote the denominators of the numbers $1 / z$ and $z /(1-z)$, respectively.

In the limiting case $z \rightarrow 1$ we obtain

$$
\widetilde{r}_{n}^{\prime}(1)=\widetilde{u}_{n} \zeta(2)-\widetilde{v}_{n}, \quad \widetilde{r}_{n}^{\prime \prime}(1)=\widetilde{u}_{n} \zeta(3)-\widetilde{w}_{n}, \quad n=0,1, \ldots
$$

where for $\widetilde{u}_{n}=\widetilde{u}_{n}(1), \widetilde{v}_{n}=\widetilde{v}_{n}(1)$, and $\widetilde{w}_{n}=\widetilde{w}_{n}(1)$ from (50), (51) we can write

$$
\widetilde{u}_{n} \in \mathbb{Z}, \quad D_{n} D_{2 n} \widetilde{v}_{n} \in \mathbb{Z}, \quad D_{n} D_{2 n}^{2} \widetilde{w}_{n} \in \mathbb{Z} .
$$

Finally, we take the rational function

$$
\widetilde{\widetilde{R}}_{n}(t)=\frac{(t-1)(t-2) \cdots(t-n) \cdot(2 t-1)(2 t-2) \cdots(2 t-n)}{(t(t+1)(t+2) \cdots(t+n))^{2}}
$$

and consider the following two series:

$$
\begin{aligned}
\left.\frac{1}{2} \sum_{\nu=1}^{\infty}(-1)^{\nu-1} R_{n}(t)\right|_{t=\nu / 2} & =\widetilde{\widetilde{u}}_{n} \zeta(2)-\widetilde{\widetilde{v}}_{n} \\
-\left.\frac{1}{2} \sum_{\nu=1}^{\infty} \frac{\mathrm{d} R_{n}(t)}{\mathrm{d} t}\right|_{t=\nu} & =\widetilde{\widetilde{u}}_{n} \zeta(3)-\widetilde{\widetilde{w}}_{n}
\end{aligned}
$$

The explicit formulae for the approximants allow us to show that

$$
\begin{gathered}
\widetilde{\widetilde{u}}_{n}=\sum_{k=0}^{n}\left(\begin{array}{l}
n \\
k
\end{array}\right)^{2}\left(\begin{array}{c}
n+k \\
n
\end{array}\right)\left(\begin{array}{c}
n+2 k \\
n
\end{array}\right) \in \mathbb{Z}, \quad \text { for } n=0,1,2, \ldots \\
D_{2 n}^{2} \widetilde{\widetilde{v}}_{n} \in \mathbb{Z}, \quad D_{n}^{3} \widetilde{\widetilde{w}}_{n} \in \mathbb{Z},
\end{gathered}
$$


Theorem 5. For $n=0,1,2, \ldots$, the following equalities are true:

$$
\left(\begin{array}{c}
2 n \\
n
\end{array}\right)^{-1} u_{n}=\widetilde{u}_{n}=\widetilde{\widetilde{u}}_{n}, \quad\left(\begin{array}{c}
2 n \\
n
\end{array}\right)^{-1} v_{n}=\widetilde{v}_{n}=\widetilde{\widetilde{v}}_{n}, \quad\left(\begin{array}{c}
2 n \\
n
\end{array}\right)^{-1} w_{n}=\widetilde{w}_{n}=\widetilde{\widetilde{w}}_{n}
$$

that is, the three hypergeometric constructions give the same sequence of simultaneous rational approximations to $1, \zeta(2)$ and $\zeta(3)$.

From Theorem 5 and the inclusions (48), (52), (53) one may easily deduce that

$$
\widetilde{u}_{n} \in \mathbb{Z}, \quad D_{n} D_{2 n} \widetilde{v}_{n} \in \mathbb{Z}, \quad D_{n}^{3} \widetilde{w}_{n} \in \mathbb{Z}, \quad \text { for } \quad n=0,1,2, \ldots
$$

Theorem 5 can be shown by means of certain hypergeometric identities. A simpler way (used in [167] and [180]) is based on the algorithm of creative telescoping. Indeed, the above sequences (54) satisfy the Apéry-type polynomial recurrence relation

$$
\begin{aligned}
& 2\left(946 n^{2}-731 n+153\right)(2 n+1)(n+1)^{3} u_{n+1} \\
& -2\left(104060 n^{6}+127710 n^{5}+12788 n^{4}-34525 n^{3}-8482 n^{2}+3298 n+1071\right) u_{n} \\
& +2\left(3784 n^{5}-1032 n^{4}-1925 n^{3}+853 n^{2}+328 n-184\right) n u_{n-1} \\
& -\left(946 n^{2}+1161 n+368\right) n(n-1)^{3} u_{n-2}=0, \quad n=2,3, \ldots,
\end{aligned}
$$

of order 3 , and the necessary initial data is as follows:

$$
\begin{gathered}
\widetilde{u}_{0}=1, \quad \widetilde{u}_{1}=7, \quad \widetilde{u}_{2}=163 \\
\widetilde{v}_{0}=0, \quad \widetilde{v}_{1}=\frac{23}{2}, \quad \widetilde{v}_{2}=\frac{2145}{8}, \quad \widetilde{w}_{0}=0, \quad \widetilde{w}_{1}=\frac{17}{2}, \quad \widetilde{w}_{2}=\frac{3135}{16} .
\end{gathered}
$$

In addition,

$$
\begin{gathered}
\limsup _{n \rightarrow \infty}\left|\widetilde{r}_{n}\right|^{1 / n}=\limsup _{n \rightarrow \infty}\left|\widetilde{r}_{n}^{\prime \prime}\right|^{1 / n}=\left|\lambda_{1,2}\right|=0.067442248 \ldots, \\
\lim _{n \rightarrow \infty}\left|\widetilde{u}_{n}\right|^{1 / n}=\lim _{n \rightarrow \infty}\left|\widetilde{v}_{n}\right|^{1 / n}=\lim _{n \rightarrow \infty}\left|\widetilde{w}_{n}\right|^{1 / n}=\lambda_{3}=54.96369509 \ldots
\end{gathered}
$$

where $\lambda_{1,2}=0.018152450 \ldots \pm i 0.064953409 \ldots$ and $\lambda_{3}$ are zeros of the characteristic polynomial $4 \lambda^{3}-220 \lambda^{2}+8 \lambda-1$.

Since $\log \left|\lambda_{1,2}\right|=-2.69648361 \ldots>-3$, from (55) and the above we cannot conclude about the irrationality of either $\zeta(2)$ or $\zeta(3)$. However, the use of an asymmetric rational function

$$
\begin{aligned}
R(t)= & R(\boldsymbol{a}, \boldsymbol{b} ; t) \\
= & \frac{\left(2 t+b_{0}\right)\left(2 t+b_{0}+1\right) \cdots\left(2 t+a_{0}-1\right)}{\left(a_{0}-b_{0}\right) !} \cdot \frac{\left(t+b_{1}\right) \cdots\left(t+a_{1}-1\right)}{\left(a_{1}-b_{1}\right) !} \\
& \quad \times \frac{\left(b_{2}-a_{2}-1\right) !}{\left(t+a_{2}\right) \cdots\left(t+b_{2}-1\right)} \cdot \frac{\left(b_{3}-a_{3}-1\right) !}{\left(t+a_{3}\right) \cdots\left(t+b_{3}-1\right)} \\
= & \frac{\left(b_{2}-a_{2}-1\right) !\left(b_{3}-a_{3}-1\right) !}{\left(a_{0}-b_{0}\right) !\left(a_{1}-b_{1}\right) !} \cdot \frac{\Gamma\left(2 t+a_{0}\right) \Gamma\left(t+a_{1}\right) \Gamma\left(t+a_{2}\right) \Gamma\left(t+a_{3}\right)}{\Gamma\left(2 t+b_{0}\right) \Gamma\left(t+b_{1}\right) \Gamma\left(t+b_{2}\right) \Gamma\left(t+b_{3}\right)},
\end{aligned}
$$


where the integers $\boldsymbol{a}$ and $\boldsymbol{b}$ satisfy

$$
\begin{gathered}
b_{1}=1<a_{1}, a_{2}, a_{3}<b_{2}, b_{3}, \quad b_{0}<a_{0} \leqslant 2 \max \left\{a_{1}, a_{2}, a_{3}\right\}, \\
a_{0}+a_{1}+a_{2}+a_{3} \leqslant b_{0}+b_{1}+b_{2}+b_{3}+2,
\end{gathered}
$$

lead to the following curious application.

Taking

$$
\begin{gathered}
a_{0}=10 n+\frac{1}{2}, \quad a_{1}=6 n+1, \quad a_{2}=7 n+1, \quad a_{3}=8 n+1, \\
b_{0}=6 n+1, \quad b_{1}=1, \quad b_{2}=13 n+2, \quad b_{3}=12 n+2,
\end{gathered}
$$

for the coefficients of linear forms

$$
\begin{aligned}
& r_{n}=\left.\sum_{\nu=-10 n}^{\infty}(-1)^{\nu} R(t)\right|_{t=\nu / 2}=u_{n} \zeta(2)-v_{n}, \\
& r_{n}^{\prime}=\left.\sum_{\nu=-5 n}^{\infty} \frac{\mathrm{d} R(t)}{\mathrm{d} t}\right|_{t=\nu}=u_{n} \zeta(3)-w_{n},
\end{aligned}
$$

we obtain the inclusions

$$
\Phi_{n}^{-1} u_{n} \in \mathbb{Z}, \quad D_{8 n} D_{16 n} \Phi_{n}^{-1} v_{n} \in \mathbb{Z}, \quad D_{8 n}^{3} \Phi_{n}^{-1} w_{n} \in \mathbb{Z},
$$

where $\Phi_{n}$ is a certain product over primes,

$$
\lim _{n \rightarrow \infty} \frac{\log \Phi_{n}}{n}=8.48973583 \ldots
$$

On the other hand,

$$
\limsup _{n \rightarrow \infty} \frac{\log \left|r_{n}\right|}{n}=\limsup _{n \rightarrow \infty} \frac{\log \left|r_{n}^{\prime}\right|}{n}=-17.610428885 \ldots
$$

Thus, the linear forms $r_{n}$ and $r_{n}^{\prime}$ allow one to deduce the irrationality of either $\zeta(2)$ or $\zeta(3)$, but not to obtain their simultaneous $\mathbb{Q}$-linear independence with 1 (the common denominator of the coefficients is $\left.D_{8 n}^{2} D_{16 n} \Phi_{n}^{-1}\right)$.

1.4. $q$-Analogues of zeta values. It is customary to call $q$-dependent quantities, which become ordinary objects as $q \rightarrow 1$ (at least formally), q-analogues or $q$-etensions. A possible way to $q$-extend the values of Riemann's zeta function reads as follows (here $q \in \mathbb{C},|q|<1$ ):

$$
\zeta_{q}(s)=\sum_{n=1}^{\infty} \sigma_{s-1}(n) q^{n}=\sum_{\nu=1}^{\infty} \frac{\nu^{s-1} q^{\nu}}{1-q^{\nu}}=\sum_{\nu=1}^{\infty} \frac{q^{\nu} \rho_{s}\left(q^{\nu}\right)}{\left(1-q^{\nu}\right)^{s}}, \quad s=1,2, \ldots
$$

where $\sigma_{s-1}(n)=\sum_{d \mid n} d^{s-1}$ denotes the sum of powers of the divisors, and the polynomials $\rho_{s}(x) \in \mathbb{Z}[x]$ can be defined recursively by means of the formulae

$$
\rho_{1}=1 \quad \text { and } \quad \rho_{s+1}=(1+(s-1) x) \rho_{s}+x(1-x) \frac{\mathrm{d} \rho_{s}}{\mathrm{~d} x} \quad \text { for } s=1,2, \ldots .
$$


Then we have the limiting relations

$$
\lim _{\substack{q \rightarrow 1 \\|q|<1}}(1-q)^{s} \zeta_{q}(s)=\rho_{s}(1) \cdot \zeta(s)=(s-1) ! \cdot \zeta(s), \quad s=2,3, \ldots ;
$$

the equality $\rho_{s}(1)=(s-1)$ ! follows from (57). The $q$-zeta values (56) so defined lead one to a circle of new interesting problems in the theory of diophantine approximations and transcendental numbers [168], which are extensions of relative problems for usual zeta values. It is not hard to show [170] that $\zeta_{q}(s)$ is transcendental as a function of variable $q$, and also [109] that the $q$-zeta values form a set of linearly independent over $\mathbb{C}(q)$ functions.

For even $s \geqslant 2$, the series $E_{s}(q)=1-2 s \zeta_{q}(s) / B_{s}$, where $B_{s} \in \mathbb{Q}$ are the Bernoulli numbers, are known as Eisenstein series. Therefore, the modular origin (with respect to $\tau=\frac{\log q}{2 \pi i}$; see also $\S 2.3$ below) of the functions $E_{4}, E_{6}, E_{8}, \ldots$ implies the algebraic independence of $\zeta_{q}(2), \zeta_{q}(4), \zeta_{q}(6)$ over $\mathbb{Q}[q]$, while all other even $q$-zeta values are polynomials in $\zeta_{q}(4)$ and $\zeta_{q}(6)$. In this interpretation, the consequence of Nesterenko's theorem [102], numbers $\zeta_{q}(2), \zeta_{q}(4), \zeta_{q}(6)$ are algebraically independent over $\mathbb{Q}$ for algebraic $q, 0<|q|<1$, is a complete $q$-extension of Lindemann's theorem [90], $\zeta(2)=\pi^{2} / 6$ is transcendental. Not much is known about the arithmetic nature of odd $q$-zeta values. P. Erdös [52] showed the irrationality of $\zeta_{q}(1)$ (the $q$-harmonic series) when $q=p^{-1}$ for $p \in \mathbb{Z} \backslash\{0, \pm 1\}$; other proofs of this fact are given in [27] and [32], while the works [37] and [139] contain the estimate

$$
\mu\left(\zeta_{q}(1)\right) \leqslant \frac{2 \pi^{2}}{\pi^{2}-2}=2.50828476 \ldots
$$

for the irrationality exponent of $\zeta_{q}(1)$ under the same assumptions on $q$. The construction of linear approximation forms for $\zeta_{q}(1)$ in [37] and [139] has several common features with the construction of Apéry's approximations (23), (31). This motivated W. Van Assche to formulate in [139] the problem of constructing linear approximation forms for $\zeta_{q}(2)$ and $\zeta_{q}(3)$, which demonstrate the irrationality of the numbers when $q^{-1} \in \mathbb{Z} \backslash\{0, \pm 1\}$ and which become, as $q \rightarrow 1$, Apéry's sequences $u_{n}^{\prime} \zeta(2)-v_{n}^{\prime}$ and $u_{n} \zeta(3)-v_{n}$, respectively (from $\S 1.1$ ).

The methods of investigating arithmetic properties of numbers $\zeta(s), s=2,3, \ldots$, successfully extends to $q$-zeta values. Namely, we mean the hypergeometric construction of linear forms as well as the arithmetic method accomplished by the group structure approach of G. Rhin and C. Viola [112], [113], [144]. For each of these constituents we can indicate the required $q$-extension: for example, the use of $q$-basic hypergeometric series, Heine's classical transformation [61] and the $q$-arithmetic method [164] (Table 1 contains corresponding parallels between ordinary and $q$-arithmetic) allows us in [172] to sharpen the estimate (58) for the irrationality exponent of the $q$-harmonic series: $\mu\left(\zeta_{q}(1)\right) \leqslant 2.46497868 \ldots$.

Using a $q$-analogue of the hypergeometric ${ }_{3} F_{2}(1)$-series and Hall's transformation [61], we not only solve in [165] the problem of Van Asshe for $\zeta_{q}(2)$ but also optimise the estimate for the irrationality exponent of the number.

Theorem 6. For each $q=1 / p, p \in \mathbb{Z} \backslash\{0, \pm 1\}$, number $\zeta_{q}(2)$ is irrational whose irrationality exponent satisfies the inequality

$$
\mu\left(\zeta_{q}(2)\right) \leqslant 4.07869374 \ldots
$$




\begin{tabular}{|c|c|}
\hline ordinary objects & $q$-extensions, $p=1 / q \in \mathbb{Z} \backslash\{0, \pm 1\}$ \\
\hline numbers $n \in \mathbb{Z}$ & $q$-numbers $[n]_{p}=\frac{p^{n}-1}{p-1} \in \mathbb{Z}[p]$ \\
\hline primes $l \in\{2,3,5,7, \ldots\} \subset \mathbb{Z}$ & $\begin{array}{l}\text { irreducible cyclotomic polynomials } \\
\Phi_{l}(p)=\prod_{\substack{k=1 \\
(k, l)=1}}^{l}\left(p-e^{2 \pi i k / l}\right) \in \mathbb{Z}[p]\end{array}$ \\
\hline Euler's gamma function $\Gamma(t)$ & $\begin{array}{c}\text { Jackson's q-gamma function } \\
\Gamma_{q}(t)=\frac{\prod_{\nu=1}^{\infty}\left(1-q^{\nu}\right)}{\prod_{\nu=1}^{\infty}\left(1-q^{t+\nu-1}\right)}(1-q)^{1-t}\end{array}$ \\
\hline $\begin{array}{l}\text { factorial } n !=\Gamma(n+1) \\
\qquad n !=\prod_{\nu=1}^{n} \nu \in \mathbb{Z}\end{array}$ & $\begin{array}{c}q \text {-factorial }[n]_{q} !=\Gamma_{q}(n+1) \\
{[n]_{p} !=\prod_{\nu=1}^{n} \frac{p^{\nu}-1}{p-1}=p^{n(n-1) / 2}[n]_{q} ! \in \mathbb{Z}[p]}\end{array}$ \\
\hline $\operatorname{ord}_{l} n !=\left\lfloor\frac{n}{l}\right\rfloor+\left\lfloor\frac{n}{l^{2}}\right\rfloor+\cdots$ & $\operatorname{ord}_{\Phi_{l}(p)}[n]_{p} !=\left\lfloor\frac{n}{l}\right\rfloor, \quad l=2,3,4, \ldots$ \\
\hline $\begin{aligned} D_{n} & =\operatorname{lcm}(1, \ldots, n) \\
& =\prod_{\text {primes } l \leqslant n} l^{\lfloor\log n / \log l\rfloor} \in \mathbb{Z}\end{aligned}$ & $\begin{aligned} D_{n}(p) & =\operatorname{lcm}\left([1]_{p}, \ldots,[n]_{p}\right) \\
& =\prod_{l=1}^{n} \Phi_{l}(p) \in \mathbb{Z}[p]\end{aligned}$ \\
\hline $\begin{array}{l}\text { the prime number theorem } \\
\qquad \lim _{n \rightarrow \infty} \frac{\log D_{n}}{n}=1\end{array}$ & $\begin{array}{l}\text { Mertens' formula } \\
\lim _{n \rightarrow \infty} \frac{\log \left|D_{n}(p)\right|}{n^{2} \log |p|}=\frac{3}{\pi^{2}}\end{array}$ \\
\hline
\end{tabular}

Table 1. Comparison of the $q$-arithmetic with ordinary arithmetic. Here $\lfloor\cdot\rfloor$ is the integral part of a number and abbreviation ' $\mathrm{lcm}$ ' is used for the least common multiple

Quantitative estimates of type (59) for $\zeta_{q}(2)$ (which show that the number is the non-Liuovillian for $q^{-1} \in \mathbb{Z} \backslash\{0, \pm 1\}$ ) were not known before, although as mentioned earlier the irrationality [49] and even the transcendence of $\zeta_{q}(2)$ for any algebraic $q$ satisfying $0<|q|<1$ follows from Nesterenko's theorem [102]. A different interpretation of the rational approximations to $\zeta_{q}(2)$ in [131] allowed the authors to simplify the arithmetic part and to sharpen the estimate (59):

$$
\mu\left(\zeta_{q}(2)\right) \leqslant \frac{10 \pi^{2}}{5 \pi^{2}-24}=3.89363887 \ldots
$$

A particular case of the hypergeometric construction from [165], [131], namely,

$$
U_{n}(q) \zeta_{q}(2)-V_{n}(q)=\left.(-1)^{n} \sum_{\nu=1}^{\infty} \frac{\prod_{j=1}^{n}\left(1-q^{j}\right) \cdot \prod_{j=1}^{n}\left(1-q^{j} T\right)}{\prod_{j=0}^{n}\left(1-q^{n+1+j} T\right)^{2}} T^{n+1}\right|_{T=q^{\nu}}
$$

leads to the irrationality of $\zeta_{q}(2)$ in case of $q^{-1} \in \mathbb{Z} \backslash\{0, \pm 1\}$, while in the limit $q \rightarrow 1$ one obtains the rational approximations of Apéry $(31)$ to $\zeta(2)$. In the 
joint paper [84] we indicate a $q$-analogue of the sequence of the rational approximations (23), (24); it however does not result in the irrationality of the quantity $\zeta_{q}(3)$.

Application of the $q$-arithmetic method and the hypergeometric construction allows one to deduce other quantitative and qualitative results for $q$-zeta values. Thus, the work [84] establishes the result on the infiniteness of irrational numbers in the set of odd $q$-zeta values (a $q$-analogue of Rivoal's theorem) when $q^{-1} \in$ $\mathbb{Z} \backslash\{0, \pm 1\}$; the quantitative results of [84] were slightly improved in the recent works [58], [78]. A special status is given to the linear independence (under the same assumptions on $q$ ) of $\zeta_{q}(1), \zeta_{q}(2)$ and 1 over $\mathbb{Q}$, in both qualitative and quantitative forms; see the papers [38], [107], [138], [178] on this direction.

We also notice that one of approaches to the Riemann hypothesis (see [28]) and to integrality problems occurring in string theory (see [45] and $\S 2.2$ below) exploits integer-valued factorial quotients and the corresponding generating hypergeometric series. As shown in [146] (hypothetically in most of the cases), the $q$-counterpart of this approach leads to $q$-polynomials with non-negative coefficients.

1.5. Lower bound for $\left\|(\mathbf{3} / \mathbf{2})^{k}\right\|$ and Waring's problem. We do not aim at covering all possible applications of hypergeometric constructions in arithmetic. Our finale in Section 1 is one more problem on the border of diophantine and analytic number theories. On first sight, it might seem that the problem is not related to Apéry's theorem, but the efficient methods of its solutions, a hypergeometric construction and the arithmetic method (used, for example, in the proof of Theorems 2 and 6), convince of the opposite.

Let $\lfloor\cdot\rfloor$ and $\{\cdot\}$ denote the integer and fractional parts of a number, respectively. It is known [143] that the inequality $\left\{(3 / 2)^{k}\right\} \leqslant 1-(3 / 4)^{k}$ for $k \geqslant 6$ implies the explicit formula $g(k)=2^{k}+\left\lfloor(3 / 2)^{k}\right\rfloor-2$ for the least integer $g=g(k)$ such that every positive integer can be expressed as a sum of at most $g$ positive $k$ th powers (Waring's problem). K. Mahler [92] used Ridout's extension of Roth's theorem to show that the inequality $\left\|(3 / 2)^{k}\right\| \leqslant C^{k}$, where $\|x\|=\min (\{x\}, 1-\{x\})$ is the distance from $x \in \mathbb{R}$ to the nearest integer, has finitely many solutions in integers $k$ for any $C<1$. The particular case $C=3 / 4$ gives one the above value of $g(k)$ for all $k \geqslant K$, where $K$ is a certain absolute but ineffective constant. This motivates the question about nontrivial (that is, $C>1 / 2$ ) and effective (in terms of $K$ ) estimate of the form

$$
\left\|\left(\frac{3}{2}\right)^{k}\right\|>C^{k} \quad \text { for all } \quad k \geqslant K .
$$

The first progress towards the problem belongs to A. Baker and J. Coates [14]; by applying effective estimates of linear forms in logarithms in the $p$-adic case, they showed the validity of $(60)$ with $C=2^{-\left(1-10^{-64}\right)}$. F. Beukers [22] significantly improved on this result by showing that inequality (60) is valid with $C=2^{-0.9}=$ $0.5358 \ldots$ for $k \geqslant K=5000$ (although his proof yielded the better choice $C=$ $0.5637 \ldots$ if one did not require an explicit evaluation of the effective bound for $K$ ). Beukers' proof relied on explicit Padé approximations to a tail of the binomial series $(1-z)^{m}=\sum_{n=0}^{m}\left(\begin{array}{c}m \\ n\end{array}\right)(-z)^{n}$ and was later used by A. Dubickas [48] and L. Habsieger [74] to derive inequality (60) with $C=0.5769$ and 0.5770 , respectively. 
The latter work also includes the estimate $\left\|(3 / 2)^{k}\right\|>0.57434^{k}$ for $k \geqslant 5$ using computations from [46] and [85].

By modifying Beukers' construction [22], namely, considering Padé approximations to a tail of the series

$$
\frac{1}{(1-z)^{m+1}}=\sum_{n=0}^{\infty}\left(\begin{array}{c}
m+n \\
m
\end{array}\right) z^{n}
$$

and evaluating the explicit $p$-adic order of the binomial coefficients involved, we prove in [179] the inequality

$$
\left\|\left(\frac{3}{2}\right)^{k}\right\|>0.5803^{k}=2^{-k \cdot 0.78512916 \ldots} \quad \text { for } \quad k \geqslant K,
$$

where $K$ is a certain effective constant.

The construction in [179] allowed us to also establish the estimates

$$
\begin{aligned}
& \left\|\left(\frac{4}{3}\right)^{k}\right\|>0.4914^{k}=3^{-k \cdot 0.64672207 \ldots} \quad \text { for } \quad k \geqslant K_{1}, \\
& \left\|\left(\frac{5}{4}\right)^{k}\right\|>0.5152^{k}=4^{-k \cdot 0.47839775 \ldots} \quad \text { for } \quad k \geqslant K_{2},
\end{aligned}
$$

where $K_{1}, K_{2}$ are effective constants. The best known result for general sequences $\left\|(1+1 / N)^{k}\right\|$ is due to M. Bennett [17]: $\left\|(1+1 / N)^{k}\right\|>3^{-k}$ for $4 \leqslant N \leqslant k 3^{k}$. Our lower bound for $\left\|(4 / 3)^{k}\right\|$ complements Bennett's result [18] on the order of the additive basis $\left\{1, N^{k},(N+1)^{k},(N+2)^{k}, \ldots\right\}$ for $N=3$ (case $N=2$ corresponds to the classical Waring's problem); to solve this problem one needs the bound $\left\|(4 / 3)^{k}\right\|>(4 / 9)^{k}$ for $k \geqslant 6$. The question of effectivisation of the estimates (62) is discussed in [110].

\section{Calabi-Yau differential equations}

2.1. Arithmetic differential equations of order 2 and 3. Certain differential equations look better than others, at least arithmetically. To illustrate this principle, consider the differential equation

$$
\left(\theta^{2}-z\left(11 \theta^{2}+11 \theta+3\right)-z^{2}(\theta+1)^{2}\right) y=0, \quad \text { where } \quad \theta=z \frac{\mathrm{d}}{\mathrm{d} z} .
$$

What is special about it? First of all, it has a unique analytic solution $y_{0}(z)=f(z)$ with $f(0)=1$; another solution may be given in the form $y_{1}(z)=f(z) \log z+g(z)$ with $g(0)=0$. Secondly, the coefficients in the Taylor expansion $f(z)=\sum_{n=0}^{\infty} A_{n} z^{n}$ are integral, $f(z) \in 1+z \mathbb{Z}[[z]]$, which can be hardly seen from the defining recurrence

$$
(n+1)^{2} A_{n+1}-\left(11 n^{2}+11 n+3\right) A_{n}-n^{2} A_{n-1}=0 \quad \text { for } n=0,1, \ldots, \quad A_{0}=1
$$

(cf. (25)), but follows from the explicit expression

$$
A_{n}=\sum_{k=0}^{n}\left(\begin{array}{l}
n \\
k
\end{array}\right)^{2}\left(\begin{array}{c}
n+k \\
n
\end{array}\right), \quad n=0,1, \ldots,
$$


due to R. Apéry [12]; note that these numbers appear in Apéry's proof of the irrationality of $\zeta(2)$. Thirdly, the expansion $q(z)=\exp \left(y_{1}(z) / y_{0}(z)\right)=z \exp (g(z) / f(z))$ also has integral coefficients, $q(z) \in z \mathbb{Z}[[z]]$. This follows from the fact that the functional inverse $z(q)$,

$$
z(q)=q \prod_{n=1}^{\infty}\left(1-q^{n}\right)^{5\left(\frac{n}{5}\right)},
$$

where $\left(\frac{n}{5}\right)$ denotes the Legendre symbol, lies in $q \mathbb{Z}[[q]]$. The formula in (66), due to F. Beukers [23], shows that $z(q)$ is a modular function with respect to the congruence subgroup $\Gamma_{1}(5)$ of $\mathrm{SL}_{2}(\mathbb{Z})$.

If the reader is not so much surprised by these integrality properties, then try to find more such cases, replacing the differential operator in (63) by the more general one

$$
D=D(a, b, c):=\theta^{2}-z\left(a \theta^{2}+a \theta+b\right)+c z^{2}(\theta+1)^{2} .
$$

To ensure the required integrality one easily gets $a, b, c \in \mathbb{Z}$, but for a generic choice of the parameters already the second feature $\left(y_{0}(z)=f(z) \in 1+z \mathbb{Z}[[z]]\right)$ fails 'almost always'. In fact, this problem was studied by F. Beukers [25] and D. Zagier [154]. The exhaustive experimental search in [154] resulted in 14 (non-degenerate) examples of the triplets $(a, b, c) \in \mathbb{Z}^{3}$ when both this and the third property (the integrality of the corresponding expansion $z(q)$ ) happen; the latter follows from modular interpretations of $z(q)$.

\begin{tabular}{|c|c|c|}
\hline$\#$ in [5] & $\#$ in $[154]$ & $(a, b, c)$ \\
\hline$(\mathrm{A})$ & $\# 11$ & $(16,4,0)$ \\
(B) & $\# 14$ & $(27,6,0)$ \\
(C) & $\# 20$ & $(64,12,0)$ \\
$(\mathrm{D})$ & & $(432,60,0)$ \\
(e) & $\# 19$ & $\left(32,12,16^{2}\right)$ \\
(h) & $\# 25$ & $\left(54,21,27^{2}\right)$ \\
$(\mathrm{i})$ & $\# 26$ & $\left(128,52,64^{2}\right)$ \\
$(\mathrm{j})$ & & $\left(864,372,432^{2}\right)$ \\
(a) & $\# 5, \mathbf{A}$ & $(7,2,-8)$ \\
(b) & $\# 9, \mathbf{D}$ & $(11,3,-1)$ \\
(c) & $\# 8, \mathbf{C}$ & $(10,3,9)$ \\
(d) & $\# 10, \mathbf{E}$ & $(12,4,32)$ \\
(f) & $\# 7, \mathbf{B}$ & $(9,3,27)$ \\
(g) & $\# 13, \mathbf{F}$ & $(17,6,72)$ \\
\hline
\end{tabular}

\begin{tabular}{|c|c|}
\hline$\#$ in $[5]$ & $(\hat{a}, \hat{b}, \hat{c})$ \\
\hline$(\beta)$ & $\left(16,8,16^{2}\right)$ \\
$(\iota)$ & $\left(27,15,27^{2}\right)$ \\
$(\vartheta)$ & $\left(64,40,64^{2}\right)$ \\
$(\kappa)$ & $\left(432,312,432^{2}\right)$ \\
& $(32,8,0)$ \\
& $(54,12,0)$ \\
& $(128,24,0)$ \\
$(\delta)$ & $(864,120,0)$ \\
$(\eta)$ & $(7,3,81)$ \\
$(\alpha)$ & $(11,5,125)$ \\
$(\epsilon)$ & $(12,4,64)$ \\
$(\zeta)$ & $(9,3,-27)$ \\
$(\gamma)$ & $(17,5,1)$ \\
\hline
\end{tabular}

Table 2. Arithmetic differential operators $D(a, b, c)$ and $\hat{D}(\hat{a}, \hat{b}, \hat{c})$

A natural extension of the above problem to 3rd order linear differential equations is prompted by the other Apéry's sequence used in his proof [12] of the irrationality of $\zeta(3)$. One takes the family of differential operators

$$
\hat{D}=\hat{D}(\hat{a}, \hat{b}, \hat{c}):=\theta^{3}-z(2 \theta+1)\left(\hat{a} \theta^{2}+\hat{a} \theta+\hat{b}\right)+\hat{c} z^{2}(\theta+1)^{3}
$$

and looks for the cases when the two solutions $f(z) \in 1+z \mathbb{C}[[z]]$ and $f(z) \log z+$ $g(z)$ with $g(0)=0$ of the corresponding differential equation satisfy $f(z) \in \mathbb{Z}[[z]]$ 
and $\exp (g(z) / f(z)) \in \mathbb{Z}[[z]]$. Apart from some degenerate cases, we have found in [6] again 14 triplets $(\hat{a}, \hat{b}, \hat{c}) \in \mathbb{Z}^{3}$ meeting the integrality conditions; the second property holds in all these cases as a modular bonus. Apéry's example corresponds to the case $(\hat{a}, \hat{b}, \hat{c})=(17,5,1)$. Table 2 lists the corresponding 14 examples of order 2 and 14 examples of order 3 , while the following theorem indicates an explicit relation between them. The proof of the theorem as well as a geometric motivation for it can be found in [5; Theorem 1]; there explicit binomial expressions for the analytic solutions $f(z)$ are given as well.

Theorem 7. Let the triplets $(a, b, c)$ and $(\hat{a}, \hat{b}, \hat{c})$ be related by the formulae

$$
\hat{a}=a, \quad \hat{b}=a-2 b \quad \text { and } \quad \hat{c}=a^{2}-4 c .
$$

For the differential operators $D$ and $\widehat{D}$ given in (67) and (68), denote by $f(z)$ and $\hat{f}(z)$ the analytic solutions of $D y=0$ and $\widehat{D} y=0$, respectively, with $f(0)=\hat{f}(0)=$ 1. Then

$$
f(z)^{2}=\frac{1}{1-a z+c z^{2}} \hat{f}\left(\frac{-z}{1-a z+c z^{2}}\right) .
$$

2.2. Arithmetic differential equations of order 4 and 5. How can one generalise the above problem of finding 'arithmetically nice' linear differential equations (operators)? An approach we followed in [6], [3], at least up to order 5, was not specifying the form of the operator, like in (67) and (68), but posing the following:

(i) the differential equation is of Fuchsian type, that is, all its singular points are regular; in addition, the local exponents at $z=0$ are zero;

(ii) the unique analytic solution $y_{0}(z)=f(z)$ with $f(0)=1$ at the origin have integral coefficients, $f(z) \in 1+z \mathbb{Z}[[z]]$; and

(iii) the solution $y_{1}(z)=f(z) \log z+g(z)$ with $g(0)=0$ gives rise to the integral expansion $q(z):=\exp \left(y_{1}(z) / y_{0}(z)\right) \in z \mathbb{Z}[[z]]$.

Requirement (i), known as the condition of maximally unipotent monodromy (MUM), means that the corresponding differential operator written as a polynomial in variable $z$ with coefficients from $\mathbb{C}[[\theta]]$ has constant term $\theta^{m}$, where $m$ is the order - degree in $\theta$; the local monodromy around 0 consists of a single Jordan block of maximal size. Note that (i) guarantees the uniqueness of the above $y_{0}(z)$ and $y_{1}(z)$. Condition (ii) can be usually relaxed to $f(C z) \in 1+z \mathbb{Z}[[z]]$ for some positive integer $C$ (without the scaling $z \mapsto C z$, many of the resulting formulae look 'more natural'). Property (iii) implies that the functional inverse $z(q)$, the so-called mirror map, also has integral expansion; furthermore, one consider $q$ as a new variable, at least in a neighbourhood of the origin.

In fact, in [6], [3] we posed on 4 th order differential equations ${ }^{1}$

$$
y^{\prime \prime \prime \prime}+P y^{\prime \prime \prime}+Q y^{\prime \prime}+R y^{\prime}+S y=0
$$

some extra conditions as well:

(iv) the 'Calabi-Yau' or 'self-duality' condition

$$
R=\frac{1}{2} P Q-\frac{1}{8} P^{3}+Q^{\prime}-\frac{3}{4} P P^{\prime}-\frac{1}{2} P^{\prime \prime},
$$

which determines the structure of the projective monodromy group; and

\footnotetext{
${ }^{1}$ Throughout this chapter we use the prime' for $z$-derivatives.
} 
(v) the integrality of a related sequence of numbers $N_{0}, N_{1}, \ldots$, known as instanton numbers in the physics literature; these arise as coefficients in the Lambert $q$-expansion of the so-called Yukawa coupling

$$
\begin{aligned}
K & =K(q):=\frac{N_{0}}{y_{0}^{2}}\left(q \frac{\mathrm{d} q}{\mathrm{~d} z}\right)^{3} \exp \left(-\frac{1}{2} \int P(z) \mathrm{d} z\right) \\
& =N_{0}+\sum_{d=1}^{\infty} \frac{N_{d} d^{3} q^{d}}{1-q^{d}} .
\end{aligned}
$$

For a long time we have been confident that in all examples these additional conditions (iv), (v) are satisfied automatically when (i)-(iii) hold. However we have learnt recently from M. Bogner and S. Reiter [29] that the differential operator

$$
\theta^{4}-8 z(2 \theta+1)^{2}\left(5 \theta^{2}+5 \theta+2\right)+192 z^{2}(2 \theta+1)(2 \theta+3)(3 \theta+2)(3 \theta+4)
$$

satisfies conditions (i)-(iv) while condition (v) seems to fail. Therefore, the CalabiYau equations of order 4 are characterised by all conditions (i)-(v). Furthermore, the antisymmetric square of any 4th order Calabi-Yau equation is a linear differential equation of order 5; when it meets conditions (i)-(iii) above (and it is always the case for all known examples, although this fact can be shown rigorously only for some instances), we call it a Calabi-Yau equation of order 5.

Our experimental search [6], [3] resulted in more than 400 examples of such differential equations. The corresponding differential operators are of Calabi-Yau type, since some of these examples can be identified with Picard-Fuchs differential equations for the periods of 1-parameter families of Calabi-Yau manifolds. For an entry in our table from [3], checking (i) and (iv) is trivial, (ii) usually follows from an explicit form of the coefficients of $f(z)$ (when it is available), while (iii) can be verified in certain cases using some of Dwork's $p$-adic techniques. Substantial progress in the latter direction was obtained recently by C. Krattenthaler and T. Rivoal [82], [83].

Basic examples of Calabi-Yau differential equations are given by the general hypergeometric differential equation (2) of order $m=4$ (and $m=5$ ) satisfied by the hypergeometric series (1). The equation (2) has (smallest possible) degree 1 in $z$ and condition (i) forces $b_{2}=\cdots=b_{m}=1$ to hold. This motivates counting the Calabi-Yau equations and their analytic solutions as a natural arithmetic generalisation of hypergeometric equations and series.

Standard conjectures (see, e.g., [7]) predict that all Calabi-Yau differential operators in [3] should be of 'geometric origin', which means that they correspond (as subquotients of local systems) to factors of Picard-Fuchs equations satisfied by period integrals for some family of varieties over the projective line.

The work [5] contains many explicit algebraic transformations between CalabiYau differential equations and their solutions which can be thought of as higherdimensional generalisations of Theorem 7; examples are the transformation (3) from the introduction and the transformation (98) below. In [5] a simple recipe is given to diagnose when two Calabi-Yau equations are related by an algebraic transformation, as well as to write down the corresponding transformation explicitly.

Another arithmetic feature of Calabi-Yau differential equations, which was first addressed in [4] in its full generality, is calculation of the corresponding Apéry limits. 
This notion originates from Apéry's work on the irrationality of $\zeta(2)$ and $\zeta(3)$ which we review in $\S 1.1$, and is discussed in several papers in relation with 2 nd and $3 \mathrm{rd}$ order arithmetic differential equations and their modular parameterizations; the basic references are [23], [24], [150] and [154]. A way to define the Apéry limit for a given Calabi-Yau differential operator $D$ is as follows. Consider the analytic solution $y_{0}(z)=f(z) \in 1+z \mathbb{Z}[[z]]$ of the equation $D y=0$ and the (unique) analytic solution $\tilde{f}(z) \in z+z^{2} \mathbb{Q}[[z]]$ of the related inhomogeneous differential equation $D \tilde{y}=z$, and write the corresponding expansions

$$
f(z)=1+\sum_{n=1}^{\infty} u_{n} z^{n} \quad \text { and } \quad \tilde{f}(z)=z+\sum_{n=2}^{\infty} v_{n} z^{n} .
$$

Then the Apéry limit $\operatorname{Ap}(D)$ is defined by

$$
\operatorname{Ap}(D):=\lim _{n \rightarrow \infty} \frac{v_{n}}{u_{n}} .
$$

The notion is motivated by the fact that Apéry's results (25)-(29) and (16)-(21) imply

$$
\operatorname{Ap}(D(11,3,-1))=\frac{\zeta(2)}{5} \quad \text { and } \quad \operatorname{Ap}(\hat{D}(17,5,1))=\frac{\zeta(3)}{6}
$$

(cf. Table 2). It was observed in [4], with many examples proved there and in [150], that the Apéry limits of Calabi-Yau differential operators happen to be the values of certain $L$-series attached to quadratic characters and elliptic curves.

2.3. The family of Calabi-Yau quintics. In this section we review some basic geometry hidden behind the Calabi-Yau differential equations.

Let $M_{z}$ be a family of Calabi-Yau threefolds parameterized by a complex variable $z \in \mathbb{P}^{1}(\mathbb{C})$. Then periods of the unique holomorphic differential 3-form on $M_{z}$ satisfy a linear differential equation, called the Picard-Fuchs differential equation of $M_{z}$. When the Hodge number $h^{2,1}$ is equal to 1, the Picard-Fuchs differential equation has order 4 . One of the most well-known examples is perhaps the family of quintic threefolds [40]

$$
x_{1}^{5}+x_{2}^{5}+x_{3}^{5}+x_{4}^{5}+x_{5}^{5}-z^{-1 / 5} x_{1} x_{2} x_{3} x_{4} x_{5}=0
$$

in $\mathbb{P}^{4}$, whose Picard-Fuchs differential equation is

$$
\theta^{4} y-5 z(5 \theta+1)(5 \theta+2)(5 \theta+3)(5 \theta+4) y=0, \quad \theta=z \frac{\mathrm{d}}{\mathrm{d} z} .
$$

This is one of the fourteen families of Calabi-Yau threefolds [3] whose Picard-Fuchs differential equations are hypergeometric. Before discussing the special features of this and other examples of very special arithmetic differential equations, let us address much simpler instances with their classical links to the theory of modular and hypergeometric functions.

It is the classical result that the solution

$$
{ }_{2} F_{1}\left(\begin{array}{c|c}
\frac{1}{2}, \frac{1}{2} & z \\
1 & z
\end{array}\right.
$$


of the Picard-Fuchs differential equation

$$
\theta^{2} y-\frac{z}{4}(2 \theta+1)^{2} y=0
$$

for the family

$$
E_{z}: y^{2}=x(x-1)(x-z)
$$

of elliptic curves (that is, of Calabi-Yau onefolds) satisfies

$$
{ }_{2} F_{1}\left(\begin{array}{c|c}
\frac{1}{2}, \frac{1}{2} & \frac{\vartheta_{2}^{4}}{\vartheta_{3}^{4}}
\end{array}\right)=\vartheta_{3}^{2}
$$

where $\vartheta_{2}(\tau)=\sum_{n \in \mathbb{Z}} e^{\pi i \tau(n+1 / 2)^{2}}$ and $\vartheta_{3}(\tau)=\sum_{n \in \mathbb{Z}} e^{\pi i \tau n^{2}}$ are modular forms of weight $1 / 2$. In other words, under a suitable setting, $z$ becomes a modular function and the holomorphic solution of the differential equation at $z=0$ becomes a modular form of weight 1 on the congruence subgroup $\Gamma(2)$ of $\mathrm{SL}_{2}(\mathbb{Z})$. Likewise, the solution

$$
{ }_{3} F_{2}\left(\begin{array}{c|c}
\frac{1}{4}, \frac{1}{2}, \frac{3}{4} & 256 z \\
1,1 &
\end{array}\right)
$$

of the Picard-Fuchs differential equation

$$
\theta^{3} y-4 z(4 \theta+1)(4 \theta+2)(4 \theta+3) y=0
$$

for the family

$$
K_{z}: x_{1}^{4}+x_{2}^{4}+x_{3}^{4}+x_{4}^{4}-z^{-1 / 4} x_{1} x_{2} x_{3} x_{4}=0
$$

of $K 3$ surfaces (that is, of Calabi-Yau twofolds) can be interpreted as a modular form of weight 2 on $\Gamma_{0}(2)$ under a suitable setting. Therefore, one might expect that the holomorphic solution of $(75)$ at $z=0$ can be interpreted as a generalised modular (or automorphic) form.

To provide some evidence to the fact that the solutions of (75) and their derivatives form a 'richer' algebraic structure, note [94], [104] that, in the classical case, the modular form or function $f(\tau)$ and its two successive derivatives $f^{\prime}(\tau)$ and $f^{\prime \prime}(\tau)$ are algebraically independent with $q=e^{2 \pi i \tau}$ over the field $\mathbb{C}(\tau)$, while all further derivatives are algebraic over the field $\mathbb{C}\left(f(\tau), f^{\prime}(\tau), f^{\prime \prime}(\tau)\right)$. For example, the ring of quasi-modular forms $\mathbb{C}\left[E_{2}(\tau), E_{4}(\tau), E_{6}(\tau)\right]$, where

$$
\begin{gathered}
E_{2}(\tau)=1-24 \sum_{n=1}^{\infty} \frac{n q^{n}}{1-q^{n}} \\
E_{4}(\tau)=1+240 \sum_{n=1}^{\infty} \frac{n^{3} q^{n}}{1-q^{n}}, \quad E_{6}(\tau)=1-504 \sum_{n=1}^{\infty} \frac{n^{5} q^{n}}{1-q^{n}}
\end{gathered}
$$

is differentially stable [111], [169]:

$$
\begin{gathered}
\dot{E}_{2}=\frac{1}{12}\left(E_{2}^{2}-E_{4}\right), \quad \dot{E}_{4}=\frac{1}{3}\left(E_{2} E_{4}-E_{6}\right), \quad \dot{E}_{6}=\frac{1}{2}\left(E_{2} E_{6}-E_{4}^{2}\right), \\
\text { where } \quad \dot{E}:=\frac{1}{2 \pi i} \frac{\mathrm{d} E}{\mathrm{~d} \tau}=q \frac{\mathrm{d} E}{\mathrm{~d} q} .
\end{gathered}
$$


The first example of a non-linear differential equation of order 7 for the Yukawa coupling was given in [89], while in [161] it was shown that no algebraic differential equation with coefficients from $\mathbb{C}(q)$ of smaller order can be given. Note that the equation from [89] is extremely lengthy, and only recently H. Movasati [99] has managed to construct a very elegant system of non-linear differential equations associated to the family of quintic threefolds (74) and its Picard-Fuchs equation (75) which resembles Ramanujan's system (77):

$$
\begin{aligned}
& \dot{T}_{0}=\frac{1}{T_{5}}\left(\frac{6}{5} T_{0}^{5}+\frac{1}{3125} T_{0} T_{3}-\frac{1}{5} T_{4}\right) \\
& \dot{T}_{1}=\frac{1}{T_{5}}\left(-125 T_{0}^{6}+T_{0}^{4} T_{1}+125 T_{0} T_{4}+\frac{1}{3125} T_{1} T_{3}\right) \\
& \dot{T}_{2}=\frac{1}{T_{5}}\left(-1875 T_{0}^{7}-\frac{1}{5} T_{0}^{5} T_{1}+2 T_{0}^{4} T_{2}+1875 T_{0}^{2} T_{4}+\frac{1}{5} T_{1} T_{4}+\frac{2}{3125} T_{2} T_{3}\right) \\
& \dot{T}_{3}=\frac{1}{T_{5}}\left(-3125 T_{0}^{8}-\frac{1}{5} T_{0}^{5} T_{2}+3 T_{0}^{4} T_{3}+3125 T_{0}^{3} T_{4}+\frac{1}{5} T_{2} T_{4}+\frac{3}{3125} T_{3}^{2}\right) \\
& \dot{T}_{4}=\frac{1}{T_{5}}\left(5 T_{0}^{4} T_{4}+\frac{1}{625} T_{3} T_{4}\right) \\
& \dot{T}_{5}=\frac{T_{6}}{T_{5}} \\
& \dot{T}_{6}=\left(-\frac{72}{5} T_{0}^{8}-\frac{24}{3125} T_{0}^{4} T_{3}-\frac{3}{5} T_{0}^{3} T_{4}-\frac{2}{1953125} T_{3}^{2}\right)+\frac{T_{6}}{T_{5}}\left(12 T_{0}^{4}+\frac{2}{625} T_{3}\right)
\end{aligned}
$$

where

$$
\dot{T}:=5 q \frac{\mathrm{d} T}{\mathrm{~d} q} .
$$

Namely, he proved in [99] the following result.

Theorem 8. A formal power series solution

$$
T_{j}=\sum_{n=0}^{\infty} t_{j, n} q^{n}, \quad j=0,1, \ldots, 6,
$$

subject to the initial conditions

$$
t_{0,0}=\frac{1}{5}, \quad t_{0,1}=24, \quad t_{4,0}=0, \quad t_{5,0}=-\frac{1}{3125}
$$

to the above system is unique. Furthermore, the quantity

$$
-\frac{\left(T_{4}-T_{0}^{5}\right)^{2}}{625 T_{5}^{3}}=5+2875 \frac{q}{1-q}+609250 \frac{2^{2} q^{2}}{1-q^{2}}+\cdots+N_{d} \frac{d^{3} q^{d}}{1-q^{d}}+\cdots
$$

is the Yukawa coupling for the family (74), and the functions (78) are algebraically independent over $\mathbb{C}$ (in fact, over $\mathbb{C}(q, \log q)$ as shown in [161]).

The argument in both [161] and [99] of showing the algebraic independence of the functions in question is relating them to the fundamental solution of the linear 
differential equation (75) and using the monodromy structure of the equation. The latter problem was addressed in several papers on the subject: it is known [26] that the Zariski closure of the projective monodromy group of (75) but also of other 4 th order differential equations is $\mathrm{Sp}_{4}(\mathbb{C})$ (this is a consequence of $(72)$ ); what is a 'nice' choice of a basis of solutions with respect to which the monodromy matrices are in $\mathrm{Sp}_{4}(\mathbb{C})$ ? A possible choice of such basis is constructed in [41] for all arithmetic hypergeometric differential equations. For example, it is shown in [41] that one can choose the symplectic matrices

$$
\left(\begin{array}{cccc}
1 & 1 & 0 & 0 \\
0 & 1 & 0 & 0 \\
5 & 5 & 1 & 0 \\
0 & -5 & -1 & 1
\end{array}\right) \text { and }\left(\begin{array}{cccc}
1 & 0 & 0 & 0 \\
0 & 1 & 0 & 1 \\
0 & 0 & 1 & 0 \\
0 & 0 & 0 & 1
\end{array}\right)
$$

as the monodromy matrices around the singular points $z=0$ and $z=1 / 3125$ of (75). Note that the group generated by (79) is contained in the congruence subgroup

$$
\left\{\gamma \in \operatorname{Sp}_{4}(\mathbb{Z}): \gamma \equiv\left(\begin{array}{cccc}
1 & * & * & * \\
0 & 1 & * & * \\
0 & 0 & 1 & 0 \\
0 & 0 & * & 1
\end{array}\right) \quad(\bmod 5)\right\}
$$

of finite index in $\operatorname{Sp}_{4}(\mathbb{Z})$, and it was discovered numerically that similar finite-index congruence subgroups of $\operatorname{Sp}_{4}(\mathbb{Z})$ contain monodromy groups for the majority of other Calabi-Yau differential equations. This observation forms grounds for asking whether one can relate the functions like (78) coming from mirror symmetry to Siegel modular forms of degree 2 or, more generally, to non-holomorphic modular forms of degree 2 . In the next section we review some partial results in this direction discussed in [151].

2.4. $\mathrm{Sp}_{4}$ modularity. Consider a Calabi-Yau differential equation of order 4 . Its projective monodromy group $\Gamma \subset \mathrm{Sp}_{4}(\mathbb{R})$ is commensurable with a discrete subgroup of $\mathrm{Sp}_{4}(\mathbb{Z})$ (of not necessarily finite index), therefore one can gather its fundamental matrix solution

$$
\left(\begin{array}{cccc}
u_{3} & u_{3}^{\prime} & u_{3}^{\prime \prime} & u_{3}^{\prime \prime \prime} \\
u_{2} & u_{2}^{\prime} & u_{2}^{\prime \prime} & u_{2}^{\prime \prime \prime} \\
u_{1} & u_{1}^{\prime} & u_{1}^{\prime \prime} & u_{1}^{\prime \prime \prime} \\
u_{0} & u_{0}^{\prime} & u_{0}^{\prime \prime} & u_{0}^{\prime \prime \prime}
\end{array}\right)
$$

in such a way that the basis $u_{0}, u_{1}, u_{2}, u_{3}$ satisfies

$$
W\left(u_{0}, u_{2}\right)+W\left(u_{1}, u_{3}\right)=0
$$

and the monodromy matrices are in $\Gamma$. Here the notation $W\left(u_{0}, u_{1}\right):=u_{0} u_{1}^{\prime}-u_{0}^{\prime} u_{1}$ stands for the wronskian. Introduce the functions

$$
w_{j l}=C z W\left(u_{j}, u_{l}\right)=C z\left(u_{j} u_{l}^{\prime}-u_{j}^{\prime} u_{l}\right), \quad w_{j l}=-w_{l j}, \quad 0 \leqslant j, l \leqslant 3,
$$

where $C \neq 0$ is a certain normalization constant. Thanks to (80) we have a linear relation $w_{02}+w_{13}=0$; there is also a quadratic relation

$$
w_{01} w_{23}+w_{02} w_{13}+w_{03} w_{12}=0,
$$


which is tautological in terms of the $u_{j}$ s. The five linearly independent functions

$$
w_{01}, \quad w_{02}=-w_{13}, \quad w_{03}, \quad w_{12}, \quad w_{23}
$$

form a solution to a fifth order linear differential equation (the so-called antisymmetric square) with the monodromy conjugate to a subgroup commensurable to a discrete subgroup of $O_{5}(\mathbb{Z}) \simeq \operatorname{Sp}_{4}(\mathbb{Z})$. This establishes the correspondence between Calabi-Yau differential equations of order 4 and 5 .

If we now define the functions

$$
\tau_{1}(z):=\frac{w_{03}}{w_{01}}, \quad \tau_{2}(z):=\frac{w_{02}}{w_{01}}=\frac{-w_{13}}{w_{01}}, \quad \tau_{3}(z):=\frac{-w_{12}}{w_{01}},
$$

and collect them in the symmetric matrix

$$
\mathrm{T}:=\left(\begin{array}{cc}
\tau_{1} & \tau_{2} \\
\tau_{2} & \tau_{3}
\end{array}\right), \quad \operatorname{det} \mathrm{T}=\frac{w_{23}}{w_{01}},
$$

then it is routine to verify that monodromy matrices $\gamma \in \Gamma$ define the standard $\mathrm{Sp}_{4}$-action on $\mathrm{T}$ :

$$
\gamma: \mathrm{T} \mapsto(A \mathrm{~T}+B)(C \mathrm{~T}+D)^{-1}=\gamma \mathrm{T} \quad \text { for } \quad \gamma=\left(\begin{array}{ll}
A & B \\
C & D
\end{array}\right) \in \Gamma .
$$

In this record, $A, B, C$ and $D$ are $2 \times 2$ components of the $4 \times 4$ matrix $\gamma$ from $\operatorname{Sp}_{4}(\mathbb{R})$. Note that the differential Galois theory [161] implies the algebraic independence of the three entries $(81)$ of $\mathrm{T}=\mathrm{T}(z)$ over $\mathbb{C}(z)$.

The multivalued function $\tau:=\tau_{1}(z)$ takes values in a certain domain $H \subset \mathbb{C}$. Viewing $\mathrm{T}$ as a matrix-valued function of $\tau$, we say that a function $f(\mathrm{~T}(\tau)): H \rightarrow \mathbb{C}$ is a $\Gamma$-modular form of weight $k$ if

$$
f(\gamma \mathrm{T})=\operatorname{det}(C \mathrm{~T}+D)^{k} \cdot f(\mathrm{~T}) \quad \text { for all } \quad \gamma=\left(\begin{array}{cc}
A & B \\
C & D
\end{array}\right) \in \Gamma .
$$

This definition is motivated by the facts [151] that the inverse $z=z(\mathrm{~T}(\tau))$ of the map $\tau=\tau_{1}(z)$ in (81), (82) is a $\Gamma$-modular form of weight 0 , while the function $w_{01}$ viewed as a function of $\mathrm{T}=\mathrm{T}(\tau)$ is a $\Gamma$-modular form of weight 1 . Note that the $z$-derivatives of the mirror map $t(z)=u_{1}(z) / u_{0}(z)$ of the starting 4 th order Calabi-Yau equation and also of the function $\tau(z)$, which is the mirror map of the resulting 5th order Calabi-Yau equation, admit simple formulae

$$
\frac{\mathrm{d} t}{\mathrm{~d} z}=\frac{w_{01}}{C u_{0}^{2}}, \quad \frac{\mathrm{d} \tau_{1}}{\mathrm{~d} z}=\frac{u_{0}^{2}}{C g_{0} w_{01}^{2}}
$$

expressing them via the analytic solutions $u_{0}$ and $w_{01}$ of the equations. These relations in turn imply that

$$
\tau_{1}(\tau)=\tau, \quad \tau_{2}(\tau)=-\int_{0}^{\tau} t(\tau) \mathrm{d} \tau, \quad \text { and } \quad \tau_{3}(\tau)=\int_{0}^{\tau} t(\tau)^{2} \mathrm{~d} \tau,
$$

when we view $\mathrm{T}$ as a function of $\tau$. 
An unfortunate thing about the $\mathrm{Sp}_{4}$-modularity above is the fact that the imaginary part of $\mathrm{T}$ is indefinite and, thus, is not in the Siegel upper half-space. This was observed by geometric consideration in [1]; instead, M. Aganagic, V. Bouchard and A. Klemm [1] define the non-holomorphic embedding

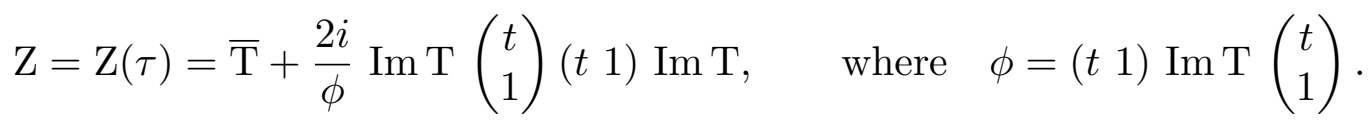

Then we indeed have $\mathrm{Z}(\tau) \in \mathbb{H}_{2}$ for all $\tau$, as well as

$$
\gamma: \mathrm{Z} \mapsto(A \mathrm{Z}+B)(C \mathrm{Z}+D)^{-1}=\gamma \mathrm{Z} \quad \text { for } \quad \gamma=\left(\begin{array}{cc}
A & B \\
C & D
\end{array}\right) \in \Gamma
$$

in addition, the function $w=\bar{\phi} \cdot w_{0}$ satisfies

$$
\gamma: w \mapsto \operatorname{det}(C \overline{\mathrm{Z}}+D) \cdot w
$$

There are at least two recipes to construct non-holomorphic modular forms $w(\mathrm{Z})$ defined on $\mathbb{H}_{2}$ which satisfy (85) for $\gamma$ from the full modular group $\operatorname{Sp}_{4}(\mathbb{Z})$ : one can use theta series attached to indefinite quadratic forms [114] or Eisenstein series [91; Chapter 18]. Below we outline a possible strategy of pulling back suitable non-holomorphic forms so constructed to the objects on the one-dimensional domain of definition, on the example of trivial Yukawa coupling and action of a finite-index subgroup $\Gamma^{\prime} \subset \mathrm{PSL}_{2}(\mathbb{Z})$ on $\tau=x+i y \in \mathbb{H}_{1}$ (that is, $y>0$ ). This case corresponds to the equality $t=\tau$ of two mirror maps.

If $t=\tau$, then formulae (84) imply

$$
\mathrm{T}(\tau)=\left(\begin{array}{cc}
\tau & -\frac{1}{2} \tau^{2} \\
-\frac{1}{2} \tau^{2} & \frac{1}{3} \tau^{3}
\end{array}\right), \quad \mathrm{Z}(x+i y)=\left(\begin{array}{cc}
x & -\frac{1}{2} x^{2} \\
-\frac{1}{2} x^{2} & \frac{1}{3} x^{3}
\end{array}\right)+\frac{i}{2}\left(\begin{array}{cc}
y & -x y \\
-x y & x^{2} y+\frac{1}{3} y^{3}
\end{array}\right)
$$

since $\phi=-\frac{4}{3} y^{3}$. We also have

$$
\operatorname{det} \mathrm{Z}=\frac{1}{12} \tau^{2}|\tau|^{2} \quad \text { and } \quad \operatorname{det} \operatorname{Im} \mathrm{Z}=\frac{1}{12} y^{4}=\frac{1}{12}(\operatorname{Im} \tau)^{4} .
$$

A slightly different version of the embedding was discovered independently by D. Zagier [153]. The related embedding of $\mathrm{SL}_{2}(\mathbb{R})$ into $\mathrm{Sp}_{4}(\mathbb{R})$,

$$
\iota:\left(\begin{array}{ll}
a & b \\
c & d
\end{array}\right) \mapsto\left(\begin{array}{cccc}
a^{2} d+2 a b c & -3 a^{2} c & a b d+\frac{1}{2} b^{2} c & \frac{1}{2} b^{2} d \\
-a^{2} b & a^{3} & -\frac{1}{2} a b^{2} & -\frac{1}{6} b^{3} \\
4 a c d+2 b c^{2} & -6 a c^{2} & a d^{2}+2 b c d & b d^{2} \\
6 c^{2} d & -6 c^{3} & 3 c d^{2} & d^{3}
\end{array}\right)
$$

shows that certain arithmetic conditions on $\Gamma^{\prime}($ for example, $b \equiv 0(\bmod 6))$ maps $\Gamma^{\prime}$ into a subgroup of $\mathrm{Sp}_{4}(\mathbb{Z})$. If we now take an arbitrary $\Gamma^{\prime}$-modular form $w_{0}(\tau)$ of weight 4 , then the corresponding function $w(x+i y)=-\frac{4}{3} y^{3} \cdot w_{0}(x+i y)$ satisfies (85) restricted to the curve

$$
\left\{\mathrm{Z}(\tau): \tau \in \mathbb{H}_{1}\right\}=\left\{\left(\begin{array}{cc}
x+\frac{i}{2} y & -\frac{1}{2} x^{2}-\frac{i}{2} x y \\
-\frac{1}{2} x^{2}-\frac{i}{2} x y & \frac{1}{3} x^{3}+\frac{i}{2} x^{2} y+\frac{i}{6} y^{3}
\end{array}\right): y>0\right\} \subset \mathbb{H}_{2} .
$$


Thus, if one starts with a function $w(\mathrm{Z})$ which obeys the transformation law (85) it is a technical issue to determine under which conditions the pullback $w_{0}(\tau)=$ $-\frac{3}{4} w(\mathrm{Z}(\tau)) /(\operatorname{Im} \tau)^{3}$ defined on $\mathbb{H}_{1}$ is holomorphic (or meromorphic) in $\mathbb{H}_{1}$.

In the general case of $t \neq \tau$ the situation seems to be more delicate because of the transcendental relation between $\tau$ and $t$. Nevertheless, knowledge of explicit power series expansions for both $\tau$ and $t$ leaves a hope to consider possible pullbacks in such cases as well.

2.5. Ramanujan-type formulae for $1 / \pi^{2}$. There is almost no mystery left about classical Ramanujan's formulae for $1 / \pi$, like (4)-(6) and their numerous generalisations; the reader is advised to consult the monograph [30] as well as the recent surveys [16] and [181]. A remarkable thing about the formulae is not only the obvious appearance of the hypergeometric series on the left-hand side but also the existence of a purely hypergeometric machinery [51], [65], [67] which enables one to prove some of these identities. This is the Wilf-Zeilberger (WZ) theory [105], [149] with the algorithm of creative telescoping as its part. Even this approach does not cover the whole variety of formulae for $1 / \pi$ (although algebraic transformations like we have in Theorem 7 significantly extend its applicability), there are some further surprising outcomes of the method. J. Guillera [65]-[68] has managed to apply the WZ theory for proving new generalisations of Ramanujan-type series, namely,

$$
\begin{gathered}
\sum_{n=0}^{\infty} \frac{\left(\frac{1}{2}\right)_{n}^{5}}{n !^{5}}\left(20 n^{2}+8 n+1\right) \frac{(-1)^{n}}{2^{2 n}}=\frac{8}{\pi^{2}}, \\
\sum_{n=0}^{\infty} \frac{\left(\frac{1}{2}\right)_{n}^{5}}{n !^{5}}\left(820 n^{2}+180 n+13\right) \frac{(-1)^{n}}{2^{10 n}}=\frac{128}{\pi^{2}}, \\
\sum_{n=0}^{\infty} \frac{\left(\frac{1}{2}\right)_{n}^{3}\left(\frac{1}{4}\right)_{n}\left(\frac{3}{4}\right)_{n}}{n !^{5}}\left(120 n^{2}+34 n+3\right) \frac{1}{2^{4 n}}=\frac{32}{\pi^{2}}, \\
\sum_{n=0}^{\infty} \frac{\left(\frac{1}{2}\right)_{n}^{3}\left(\frac{1}{3}\right)_{n}\left(\frac{2}{3}\right)_{n}}{n !^{5}}\left(74 n^{2}+27 n+3\right) \frac{3^{3 n}}{4^{3 n}}=\frac{48}{\pi^{2}} .
\end{gathered}
$$

Furthermore, this newer pattern of formulae for $1 / \pi^{2}$ suggested Guillera [66] and Guillera and Almkvist [2] to discover numerically seven additional formulae:

$$
\begin{gathered}
\sum_{n=0}^{\infty} \frac{\left(\frac{1}{2}\right)_{n}\left(\frac{1}{4}\right)_{n}\left(\frac{3}{4}\right)_{n}\left(\frac{1}{6}\right)_{n}\left(\frac{5}{6}\right)_{n}}{n !^{5}}\left(1640 n^{2}+278 n+15\right) \frac{(-1)^{n}}{2^{10 n}} \stackrel{?}{=} \frac{256 \sqrt{3}}{3 \pi^{2}} \\
\sum_{n=0}^{\infty} \frac{\left(\frac{1}{2}\right)_{n}\left(\frac{1}{4}\right)_{n}\left(\frac{3}{4}\right)_{n}\left(\frac{1}{3}\right)_{n}\left(\frac{2}{3}\right)_{n}}{n !^{5}}\left(252 n^{2}+63 n+5\right) \frac{(-1)^{n}}{48^{n}} \stackrel{?}{=} \frac{48}{\pi^{2}}, \quad(91) \\
\sum_{n=0}^{\infty} \frac{\left(\frac{1}{2}\right)_{n}\left(\frac{1}{3}\right)_{n}\left(\frac{2}{3}\right)_{n}\left(\frac{1}{6}\right)_{n}\left(\frac{5}{6}\right)_{n}}{n !^{5}}\left(5418 n^{2}+693 n+29\right) \frac{(-1)^{n}}{80^{3 n}} \stackrel{?}{=} \frac{128 \sqrt{5}}{\pi^{2}}
\end{gathered}
$$




$$
\begin{gathered}
\sum_{n=0}^{\infty} \frac{\left(\frac{1}{2}\right)_{n}\left(\frac{1}{3}\right)_{n}\left(\frac{2}{3}\right)_{n}\left(\frac{1}{6}\right)_{n}\left(\frac{5}{6}\right)_{n}}{n !^{5}}\left(1930 n^{2}+549 n+45\right) \frac{(-1)^{n} 3^{6 n}}{2^{12 n}} \stackrel{?}{=} \frac{384}{\pi^{2}} \\
\sum_{n=0}^{\infty} \frac{\left(\frac{1}{2}\right)_{n}\left(\frac{1}{3}\right)_{n}\left(\frac{2}{3}\right)_{n}\left(\frac{1}{6}\right)_{n}\left(\frac{5}{6}\right)_{n}}{n !^{5}}\left(532 n^{2}+126 n+9\right) \frac{3^{6 n}}{5^{6 n}} \stackrel{?}{=} \frac{375}{4 \pi^{2}} \\
\sum_{n=0}^{\infty} \frac{\left(\frac{1}{2}\right)_{n}\left(\frac{1}{8}\right)_{n}\left(\frac{3}{8}\right)_{n}\left(\frac{5}{8}\right)_{n}\left(\frac{7}{8}\right)_{n}}{n !^{5}}\left(1920 n^{2}+304 n+15\right) \frac{1}{7^{4 n}} \stackrel{?}{=} \frac{56 \sqrt{7}}{\pi^{2}}, \\
\sum_{n=0}^{\infty} \frac{\left(\frac{1}{2}\right)_{n}^{3}\left(\frac{1}{3}\right)_{n}\left(\frac{2}{3}\right)_{n}}{n !^{5}}\left((32-216 \phi) n^{2}+(18-162 \phi) n+(3-30 \phi)\right)(3 \phi)^{3 n} \stackrel{?}{=} \frac{3}{\pi^{2}},
\end{gathered}
$$

where

$$
\phi=\left(\frac{\sqrt{5}-1}{2}\right)^{5}=0.09016994 \ldots
$$

Note that the latter constant appears in the asymptotics of Apéry's approximations to $\zeta(2)$ (cf. (28) and (29) in $\S 1.1)$.

There exists also the ' $3 \mathrm{D}$ ' identity

$$
\sum_{n=0}^{\infty} \frac{\left(\frac{1}{2}\right)_{n}^{7}}{n !^{7}}\left(168 n^{3}+76 n^{2}+14 n+1\right) \frac{1}{2^{6 n}} \stackrel{?}{=} \frac{32}{\pi^{3}}
$$

discovered by B. Gourevich in 2002 (using an integer relations algorithm), and the most recent news is the formula

$$
\sum_{n=0}^{\infty} \frac{\left(\frac{1}{2}\right)_{n}^{7}\left(\frac{1}{4}\right)_{n}\left(\frac{3}{4}\right)_{n}}{n !^{9} 2^{12 n}}\left(43680 n^{4}+20632 n^{3}+4340 n^{2}+466 n+21\right) \stackrel{?}{=} \frac{2048}{\pi^{4}}
$$

due to J. Cullen [44].

The works [2], [69], and [151] discuss a relationship of identities (86)-(96) with the Calabi-Yau differential equations from Section 2.2. A standard example here, related to Guillera's formulae (86) and (87), is the hypergeometric series

$$
F(z)={ }_{5} F_{4}\left(\begin{array}{c}
\frac{1}{2}, \frac{1}{2}, \frac{1}{2}, \frac{1}{2}, \frac{1}{2} \\
1,1,1,1
\end{array} \mid 2^{10} z\right)=\sum_{n=0}^{\infty}\left(\begin{array}{c}
2 n \\
n
\end{array}\right)^{5} z^{n}
$$

which satisfies the 5th-order linear differential equation

$$
\left(\theta^{5}-32 z(2 \theta+1)^{5}\right) Y=0, \quad \text { where } \quad \theta=z \frac{\mathrm{d}}{\mathrm{d} z} .
$$

If $G(z)$ is another solution of the latter equation of the form $F(z) \log z+F_{1}(z)$ with $F_{1}(z) \in z \mathbb{Q}[[z]]$, then

$$
\widetilde{F}(z)=\left(1-2^{10} z\right)^{-1 / 2} \operatorname{det}\left(\begin{array}{cc}
F & G \\
\theta F & \theta G
\end{array}\right)^{1 / 2}
$$


satisfies the 4th-order equation

$$
\left(\theta^{4}-16 z\left(128 \theta^{4}+256 \theta^{3}+304 \theta^{2}+176 \theta+39\right)+2^{20} z^{2}(\theta+1)^{4}\right) Y=0,
$$

which is entry \#204 in the tables [3]. For a quadratic transformation of the new function $\widetilde{F}(z)$ one has the following explicit formula [5]:

$$
\frac{1+z}{(1-z)^{2}} \widetilde{F}\left(\frac{-z}{(1-z)^{2}}\right)=\sum_{n=0}^{\infty}\left(\sum_{k=0}^{n} 4^{n-k}\left(\begin{array}{c}
2 k \\
k
\end{array}\right)^{2}\left(\begin{array}{c}
2 n-2 k \\
n-k
\end{array}\right)\right)^{2} z^{n},
$$

where the right-hand side is the Hadamard square of the series

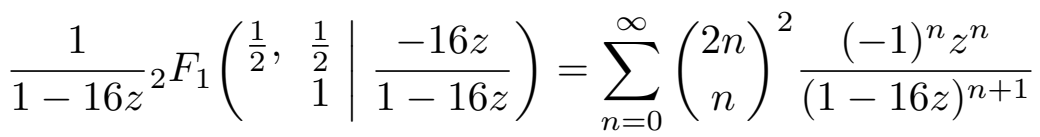

$$
\begin{aligned}
& =\sum_{n=0}^{\infty}\left(\sum_{k=0}^{n} 4^{n-k}\left(\begin{array}{c}
2 k \\
k
\end{array}\right)^{2}\left(\begin{array}{c}
2 n-2 k \\
n-k
\end{array}\right)\right) z^{n}
\end{aligned}
$$

which admits a modular parametrization. Both the 4th-order equation (97) and the differential equation (of order 4) for the right-hand side in (98) are of Calabi-Yau type. The underlying Calabi-Yau differential equations are crucial in the numerical discovery of several formulae for $1 / \pi^{2}$; the details can be found in [2].

A more 'delicate' outcome of the WZ-theoretic approach are functional identities that include the numerical evaluations as specialisations. The anticipated advantage of functional instances is their 'easier' provability because additional functional (for example, differential) equations can be used. There are several ad hoc methods in passing from numerics to functions; many of them rest on a tricky application of the Gosper-Zeilberger algorithm of creative telescoping [105].

Examples related to Ramanujan's series (4) and Guillera's series (86) can be found in [67]. Consider

$$
\begin{aligned}
& \sum_{n=0}^{\infty} \frac{\left(a+\frac{1}{2}\right)_{n}^{3}}{(a+1)_{n}^{3}}(6(n+a)+1) \cdot \frac{1}{4^{n}} \\
& \quad=\frac{4^{a+1}}{\pi \cos ^{2} \pi a} \cdot\left(\frac{\Gamma(a+1) \Gamma\left(\frac{1}{2}\right)}{\Gamma\left(a+\frac{1}{2}\right)}\right)^{3}+\frac{(4 a)^{2}}{2 a-1} \sum_{n=0}^{\infty} \frac{\left(\frac{1}{2}\right)_{n}\left(a+\frac{1}{2}\right)_{n}}{(a+1)_{n}\left(\frac{3}{2}-a\right)_{n}}
\end{aligned}
$$

and, denoting an either side of the latter expression by $f(a)$,

$$
\begin{aligned}
& \sum_{n=0}^{\infty} \frac{\left(a+\frac{1}{2}\right)_{n}^{5}}{(a+1)_{n}^{5}}\left(20(n+a)^{2}+8(n+a)+1\right) \cdot \frac{(-1)^{n}}{4^{n}} \\
& \quad=\frac{2}{\pi \cos \pi a} \cdot\left(\frac{\Gamma(a+1) \Gamma\left(\frac{1}{2}\right)}{\Gamma\left(a+\frac{1}{2}\right)}\right)^{2} f(a)+\frac{2^{5} a^{3}}{2 a-1} \sum_{n=0}^{\infty} \frac{\left(\frac{1}{2}\right)_{n}^{2}\left(a+\frac{1}{2}\right)_{n}}{(a+1)_{n}^{2}\left(\frac{3}{2}-a\right)_{n}} .
\end{aligned}
$$

The specialisation $a=0$ of (99) and (100) gives the numerical identities (4) and (86), respectively. 
Another striking arithmetic hidden in Ramanujan's formulae for $1 / \pi$ and their generalisations relies on the so-called supercongruences. It happens that if we truncate the corresponding hypergeometric series at $n=p-1$, we always get congruences modulo high powers of $p$, where $p>3$ is a prime not dividing the denominator of the argument. For example,

$$
\begin{gathered}
\sum_{n=0}^{p-1} \frac{\left(\frac{1}{2}\right)_{n}\left(\frac{1}{4}\right)_{n}\left(\frac{3}{4}\right)_{n}}{n !^{3}}(20 n+3) \frac{(-1)^{n}}{2^{2 n}} \equiv 3\left(\frac{-1}{p}\right) p \quad\left(\bmod p^{3}\right), \\
\sum_{n=0}^{p-1} \frac{\left(\frac{1}{2}\right)_{n}^{3}\left(\frac{1}{4}\right)_{n}\left(\frac{3}{4}\right)_{n}}{n !^{5}}\left(120 n^{2}+34 n+3\right) \frac{1}{2^{4 n}} \equiv 3 p^{2} \quad\left(\bmod p^{5}\right), \\
\sum_{n=0}^{p-1} \frac{\left(\frac{1}{2}\right)_{n}^{7}\left(\frac{1}{4}\right)_{n}\left(\frac{3}{4}\right)_{n}}{n !^{9} 2^{12 n}}\left(43680 n^{4}+20632 n^{3}+4340 n^{2}+466 n+21\right) \stackrel{?}{\equiv} 21 p^{4} \quad\left(\bmod p^{9}\right) .
\end{gathered}
$$

The known proofs [71], [137], [183] makes use of the Wilf-Zeilberger theory again. The work [70] discusses the general pattern for all such supercongruences, including finite analogues for 'irrational' Ramanujan-type identities like (96).

\section{Lattice sums and Mahler measures}

3.1. Dirichlet $\boldsymbol{L}$-series and Mahler measures. For a Laurent polynomial $P\left(x_{1}, \ldots, x_{n}\right)$, the Mahler measure $M(P):=e^{\mathrm{m}(P)}$, with $\mathrm{m}(P)$ defined in $(9)$, is the geometric mean of $|P|$ on the torus

$$
\mathbb{T}^{n}=\left\{\left(x_{1}, \ldots, x_{n}\right) \in \mathbb{C}^{n}:\left|x_{1}\right|=\cdots=\left|x_{n}\right|=1\right\} .
$$

Mahler's original definition [93] refers to the case $n=1$ where one has a different expression

$$
\mathrm{m}(P)=\log \left|a_{0}\right|+\sum_{j=1}^{d} \max \left\{0, \log \left|\alpha_{j}\right|\right\}
$$

for a polynomial $P(x)=a_{0} \prod_{j=1}^{d}\left(x-\alpha_{j}\right)$, as a consequence of classical Jensen's formula. For polynomials $P(x)$ with integer coefficients, clearly $\mathrm{m}(P) \geqslant 0$, with $\mathrm{m}(P)=0$ only if $P$ is monic $\left(a_{0}=1\right)$ and has all its zeros inside the unit circle (hence is a product of a monomial $x^{a}$ and a cyclotomic polynomial, by Kronecker's theorem). D. Lehmer [88] asked (already in 1933) whether $\mathrm{m}(P)$ can be arbitrary small but positive for $P(x) \in \mathbb{Z}[x]$; the smallest value he was able to find was

$\mathrm{m}\left(x^{10}+x^{9}-x^{7}-x^{6}-x^{5}-x^{4}-x^{3}+x+1\right)=\log (1.17628081 \ldots)=0.16235761 \ldots$

This still stands as the smallest positive value of $\mathrm{m}(P)$, in spite of extensive computation by D. Boyd, M. Mossinghoff and others. Although Lehmer's question is a completely different story in the study of Mahler measures, it motivated the above definition of $\mathrm{m}(P)$ to the multi-variable case because of the following limit formula proven by Boyd in 1981 [33]:

$$
\mathrm{m}\left(P\left(x, x^{N}\right)\right) \rightarrow \mathrm{m}(P(x, y)) \quad \text { as } N \rightarrow \infty .
$$


It was not realised until 1981 that the $n$-variable Mahler measure could have some 'geometric' roots. Namely, C. Smyth gave an elegant formula [33]

$$
\mathrm{m}(1+x+y)=\frac{3 \sqrt{3}}{4 \pi} L\left(\chi_{-3}, 2\right)=L^{\prime}\left(\chi_{-3},-1\right)
$$

where

$$
L\left(\chi_{-3}, s\right)=\sum_{n=1}^{\infty} \frac{\chi_{-3}(n)}{n^{s}}=1-\frac{1}{2^{s}}+\frac{1}{4^{s}}-\frac{1}{5^{s}}+\cdots
$$

is the $L$-function attached to the real odd Dirichlet character modulo 3.

The proof of Smyth's formula is worth noting here. Since $1+x+y$ is a linear function of $y$, Jensen's formula applied to one of the integrals in (9) shows that

$$
\mathrm{m}(1+x+y)=\mathrm{m}(1-x+y)=\frac{1}{2 \pi} \int_{0}^{2 \pi} \log ^{+}\left|e^{i t}-1\right| \mathrm{d} t=\frac{1}{2 \pi} \int_{0}^{2 \pi / 3} \log \left|e^{i t}-1\right| \mathrm{d} t
$$

where $\log ^{+} x=\max \{0, \log x\}$. Thus, $\mathrm{m}(1+x+y)$ is given by a special value of the Clausen integral

$$
\mathrm{Cl}_{2}(\theta)=-\int_{0}^{\theta} \log \left|e^{i t}-1\right| \mathrm{d} t=\sum_{k=1}^{\infty} \frac{\sin (k \theta)}{k^{2}}
$$

and the result follows.

A similar computation applies to many polynomials $P(x, y)=A(x) y+B(x)$ if $A(x)$ and $B(x)$ are cyclotomic and if the solutions of $|A(x)|=|B(x)|$ on $|x|=1$ are roots of unity. For example [34],

$$
\mathrm{m}(1+x+y-x y)=\frac{2}{\pi} L\left(\chi_{-4}, 2\right)=L^{\prime}\left(\chi_{-4},-1\right),
$$

where $L\left(\chi_{-4}, 2\right)=G$ is Catalan's constant (cf. (13)),

$$
\begin{aligned}
\mathrm{m}\left(1+x+x^{2}+y\right) & =\frac{3}{2} L^{\prime}\left(\chi_{-4},-1\right) \\
\mathrm{m}\left(1+x+y+x^{2} y\right) & =\frac{3}{2} L^{\prime}\left(\chi_{-3},-1\right)
\end{aligned}
$$

Later V. Maillot and J. Cassaigne [95] derived a general formula for $\mathrm{m}\left(a_{0}+a_{1} x+\right.$ $\left.a_{2} y\right)$, for arbitrary complex $a_{j}$, by means of the Bloch-Wigner dilogarithm

$$
\mathcal{D}(z)=\operatorname{Im}\left(\sum_{n=1}^{\infty} \frac{z^{n}}{n^{2}}+\log |z| \log (1-z)\right) .
$$

If $\left|a_{0}\right|,\left|a_{1}\right|$ and $\left|a_{2}\right|$ are the lengths of the sides of a planar triangle opposite the angles $\alpha_{0}, \alpha_{1}$ and $\alpha_{2}$, then

$$
\mathrm{m}\left(a_{0}+a_{1} x+a_{2} y\right)=\frac{\alpha_{0}}{\pi} \log \left|a_{0}\right|+\frac{\alpha_{1}}{\pi} \log \left|a_{1}\right|+\frac{\alpha_{2}}{\pi} \log \left|a_{2}\right|+\frac{1}{\pi} \mathcal{D}\left(\frac{\left|a_{1}\right|}{\left|a_{0}\right|} e^{i \alpha_{2}}\right)
$$


in the alternative case,

$$
\mathrm{m}\left(a_{0}+a_{1} x+a_{2} y\right)=\log \max \left\{\left|a_{0}\right|,\left|a_{1}\right|,\left|a_{2}\right|\right\} .
$$

Already this result shows a strong connection of Mahler measure evaluation with $K$-theory. That is why it is not completely mysterious to expect that more sophisticated polynomials $P(x, y)$ give rise to analogous Mahler measures expressed through special values of $L$-functions of elliptic curves. Here the counterpart to

$$
\frac{d^{3 / 2} L\left(\chi_{-d}, 2\right)}{4 \pi}=L^{\prime}\left(\chi_{-d},-1\right)
$$

is given by

$$
b_{E}=\frac{N L(E, 2)}{4 \pi^{2}}=L^{\prime}(E, 0)
$$

where $N$ is the conductor of the elliptic curve $E$ and where the latter equality is only valid if $E$ is a modular curve (that is, a smooth cubic curve over $\mathbb{Q}$ that has a rational point; the Shimura-Taniyama conjecture - the theorem now - says that all elliptic curves over $\mathbb{Q}$ are modular). In other words, there exist polynomials $P_{E}(x, y)$ for which $\mathrm{m}\left(P_{E}\right) / b_{E}$ is (presumably) rational. Without explaining deep $K$-theoretic reasons for such formulae to exist, we provide some hints on Deninger's example (12) mentioned in the introduction.

Consider

$$
P(x, y)=1+x+\frac{1}{x}+y+\frac{1}{y} .
$$

Let $x=e^{i t}$ and treat $P(x, y)$ as a polynomial in $y$ to see that

$$
|P(x, y)|=\left|1+y(1+2 \cos t)+y^{2}\right|=\left|\left(y-y_{1}(t)\right)\left(y-y_{2}(t)\right)\right|,
$$

where $y_{1}(t)=-b-\sqrt{b^{2}-1}$ with $b=b(t)=\frac{1}{2}+\cos t$. With the help of Jensen's formula,

$$
\mathrm{m}(P)=\frac{1}{\pi} \int_{0}^{\pi} \log ^{+}\left|y_{1}(t)\right| \mathrm{d} t .
$$

Since the product of the roots $y_{1}(t)$ and $y_{2}(t)$ is 1 , we will have $\left|y_{1}(t)\right|>1>\left|y_{2}(t)\right|$ exactly when the roots are real and unequal, that is, when $\cos t>\frac{1}{2}$, so $|t|<\frac{\pi}{3}$. Thus

$$
\mathrm{m}(P)=\int_{0}^{\pi / 3} \log \left(b+\sqrt{b^{2}-1}\right) \mathrm{d} t .
$$

This integral can be integrated numerically but, of course, there are various other ways to represent it, for example,

$$
\begin{aligned}
\mathrm{m}(P) & =\frac{1}{(2 \pi)^{2}} \int_{0}^{2 \pi} \int_{0}^{2 \pi} \log (1+2 \cos t+2 \cos s) \mathrm{d} t \mathrm{~d} t \\
& =\frac{1}{4} \cdot{ }_{3} F_{2}\left(\begin{array}{c}
\frac{1}{2}, \frac{1}{2}, \frac{1}{2} \\
1, \frac{3}{2}
\end{array} \mid \frac{1}{16}\right)=4 \sum_{k=0}^{\infty}\left(\begin{array}{c}
2 k \\
k
\end{array}\right)^{2} \frac{(1 / 16)^{2 k+1}}{2 k+1},
\end{aligned}
$$


the result we mentioned in (11). In a similar fashion, one can also derive a more general hypergeometric representation [120], [86], [123]

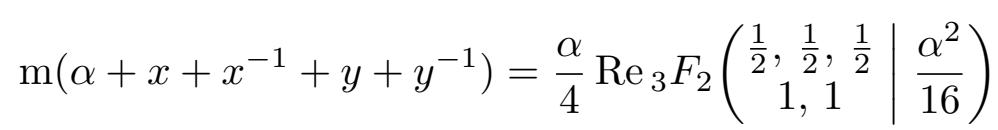

when $\alpha>0$.

On the other hand, using a cohomological interpretation of $\mathrm{m}(P(x, y))$, Deninger [47] was able to evaluate this Mahler measure as an Eisenstein-Kronecker series of the elliptic curve $E$ of conductor 15 given by

$$
1+x+\frac{1}{x}+y+\frac{1}{y}=0,
$$

and then assuming a conjecture of Beilinson, to derive that one should have

$$
\mathrm{m}(P)=r \frac{15}{(2 \pi)^{2}} L(E, 2)=r L^{\prime}(E, 0),
$$

where $r$ is a rational number (unspecified in Beilinson's conjecture). Finally, it was checked numerically that $r=1.00000000 \ldots$ (up to 200 decimal places), so that presumably (10) holds. The modularity theorem implies that, for the $L$-function $L(E, s)=\sum_{k=1}^{\infty} a_{k} k^{-s}$ attached to an elliptic curve $E$ of conductor $N$, the function $f(\tau)=\sum_{k=1}^{\infty} a_{k} q^{k}$, where $q=e^{2 \pi i \tau}$, is a cusp form for the modular group $\Gamma_{0}(N)$. In Deninger's case $N=15$, so that

$$
f(\tau)=\sum_{k=1}^{\infty} a_{k} q^{k}=q \prod_{m=1}^{\infty}\left(1-q^{m}\right)\left(1-q^{3 m}\right)\left(1-q^{5 m}\right)\left(1-q^{15 m}\right) .
$$

In view of Euler's pentagonal number formula

$$
\eta(\tau)=q^{1 / 24} \prod_{m=1}^{\infty}\left(1-q^{m}\right)=\sum_{n \in \mathbb{Z}}(-1)^{n} q^{(6 n+1)^{2} / 24}
$$

and the hypergeometric evaluation above, the final formula can be stated as (12).

It is remarkable that, in spite of the origin of formula (12), it does not involve any Mahler measure: it is a (hypergeometric) single sum evaluation of a quadruple lattice sum.

3.2. Quadruple lattice sums. Define

$$
\begin{aligned}
F(a, b, c, d) & =(a+b+c+d)^{2} \\
& \times \sum_{\substack{n_{j}=-\infty \\
j=1,2,3,4}}^{\infty} \frac{(-1)^{n_{1}+n_{2}+n_{3}+n_{4}}}{\left(a\left(6 n_{1}+1\right)^{2}+b\left(6 n_{2}+1\right)^{2}+c\left(6 n_{3}+1\right)^{2}+d\left(6 n_{4}+1\right)^{2}\right)^{2}}
\end{aligned}
$$

where the method of summation is

$$
\sum_{\substack{n_{j}=-\infty \\ j=1,2,3,4}}^{\infty}=\lim _{M \rightarrow \infty} \sum_{n_{1}=-M}^{M} \cdots \sum_{n_{4}=-M}^{M}
$$


and also set

$$
F(b, c)=F(1, b, c, b c)
$$

Many cases are known when $F(a, b, c, d)$ can be (sometimes conjecturally) reduced to a single sum, like

$$
F(3,5)=\frac{\pi^{2}}{15} \cdot{ }_{3} F_{2}\left(\begin{array}{c}
\frac{1}{2}, \frac{1}{2}, \frac{1}{2} \\
1, \frac{3}{2}
\end{array} \frac{1}{16}\right)
$$

in the case of (12). Another conjectured evaluation, due to Boyd, which is now settled in [124], is related to a conductor 20 elliptic curve and is equivalent to

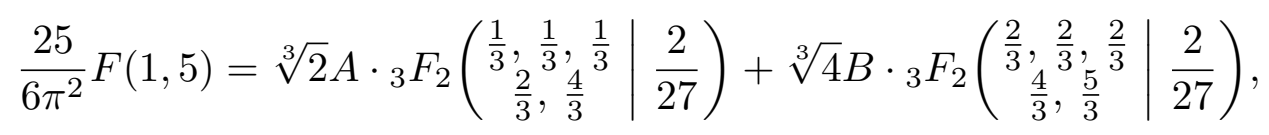

where

$$
A=\frac{\sqrt[3]{2} \Gamma\left(\frac{1}{6}\right) \Gamma\left(\frac{1}{3}\right) \Gamma\left(\frac{1}{2}\right)}{8 \sqrt{3} \pi^{2}} \text { and } \quad B=\frac{\Gamma^{3}\left(\frac{2}{3}\right)}{16 \pi^{2}} .
$$

When more general lattice sums are considered, hypergeometric functions with irrational arguments frequently appear. For instance, if $\phi=(1+\sqrt{5}) / 2$, we have

$$
\frac{225}{32 \sqrt{5} \pi^{2}} F(1,5,5,5)=\frac{A}{\sqrt[3]{\phi}} \cdot{ }_{3} F_{2}\left(\begin{array}{c}
\frac{1}{3}, \frac{1}{3}, \frac{1}{3} \\
\frac{2}{3}, \frac{4}{3}
\end{array} \mid \frac{1}{\phi}\right)+\frac{3 B}{\sqrt[3]{\phi^{2}}} \cdot{ }_{3} F_{2}\left(\begin{array}{c}
\frac{2}{3}, \frac{2}{3}, \frac{2}{3} \\
\frac{4}{3}, \frac{5}{3}
\end{array} \mid \frac{1}{\phi}\right) .
$$

While it seems likely that equations for $F(1,5,5,5)$ and $F(1,5)=F(1,1,5,5)$ both arise as special cases of formulae for $F(a, b, c, d)$, the exact nature of those formulae remains unclear.

A standard analytic strategy for proving hypergeometric evaluations is reduction of the quadruple lattice sum under consideration to a double sum and then interpreting the double sum as a special value of an elliptic function. For instance,

$$
F(1,5,5,5)=16^{2} \sum_{\substack{n=-\infty \\ k=0}}^{\infty} \frac{(-1)^{n+k}(2 k+1)}{\left((6 n+1)^{2}+15(2 k+1)^{2}\right)^{2}} .
$$

These sorts of transformations follow from well-known $q$-series results, and are quite rare. The double sum is a consequence of the following corollary to the Jacobi triple product:

$$
q^{2} \prod_{n=1}^{\infty}\left(1-q^{3 n}\right)\left(1-q^{15 n}\right)^{3}=\sum_{\substack{n=-\infty \\ k=0}}^{\infty}(-1)^{n+k}(2 k+1) q^{\left(15(2 k+1)^{2}+(6 n+1)^{2}\right) / 8}
$$

Notice that this equation gives an example of a lacunary modular form, and only in such cases the two-dimensional reduction can be achieved. The two-dimensional lattice sums are then evaluated using Ramanujan's theories of elliptic functions and modular equations [11], [19]. Several of these calculations are quite involved.

The finite amount of elliptic curves whose $L$-functions are related to four eta products is given by the following list [96]. Suppose that $E_{N}$ is an elliptic curve of conductor $N$, then $L\left(E_{N}, 2\right)=F(b, c)$ for the values of $N$ and $(b, c)$ given in Table 3 . 


\begin{tabular}{|c|c|}
\hline$N$ & $(b, c)$ \\
\hline 11 & $(1,11)$ \\
14 & $(2,7)$ \\
15 & $(3,5)$ \\
20 & $(1,5)$ \\
24 & $(2,3)$ \\
27 & $(1,3)$ \\
32 & $(1,2)$ \\
36 & $(1,1)$ \\
\hline
\end{tabular}

Table 3. Correspondence $L\left(E_{N}, 2\right)=F(b, c)$ for elliptic curves $E_{N}$ of conductor $N$

Note that case $N=11$ does not possess a known hypergeometric evaluation but is expressed through the Mahler measure,

$$
\frac{77}{4 \pi^{2}} F(1,11)=\mathrm{m}((1+x)(1+y)(1+x+y)+x y)
$$

a result originally conjectured by Boyd and recently shown by F. Brunault [36] using a $K$-theoretic argument.

Finally comes the list of hypergeometric evaluations for the corresponding lattice sums $F(b, c)=L\left(E_{N}, 2\right)$ :

$$
\begin{aligned}
& \frac{9}{2 \pi^{2}} F(1,1)=\frac{1}{9} \log 54-\frac{1}{81}{ }_{4} F_{3}\left(\begin{array}{c|c}
\frac{4}{3}, \frac{5}{3}, 1,1 & \frac{1}{2} \\
2,2,2 & 2
\end{array}\right) \\
& \frac{16}{\pi^{2}} F(1,2)=2 \log 2+\frac{1}{8}{ }_{4} F_{3}\left(\begin{array}{c|c}
\frac{3}{2}, \frac{3}{2}, 1,1 & -\frac{1}{4} \\
2,2,2 & -
\end{array}\right. \\
& \frac{8}{\pi^{2}} F(1,2)=\frac{1}{\sqrt{2}}{ }_{3} F_{2}\left(\begin{array}{c|c}
\frac{1}{2}, \frac{1}{2}, \frac{1}{2} & \frac{1}{2}, \frac{3}{2}
\end{array}\right) \\
& \frac{81}{4 \pi^{2}} F(1,3)=\log 6+\frac{1}{108}{ }_{4} F_{3}\left(\begin{array}{c|c}
\frac{4}{3}, \frac{5}{3}, 1,1 & -\frac{1}{8} \\
2,2,2 & ,
\end{array}\right.
\end{aligned}
$$

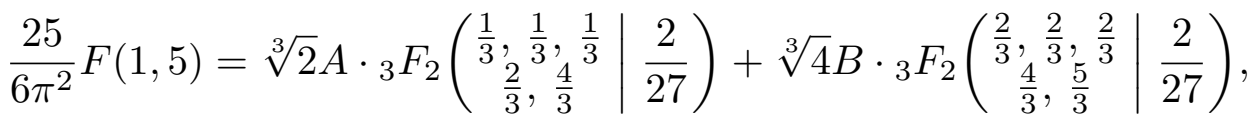

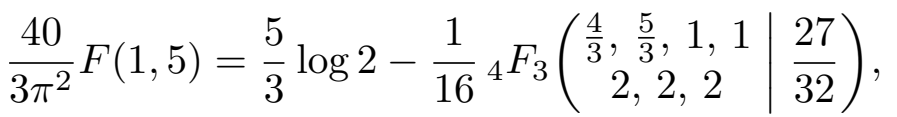

$$
\begin{aligned}
& \frac{6}{\pi^{2}} F(2,3)=\frac{1}{2}{ }_{3} F_{2}\left(\begin{array}{c|c}
\frac{1}{2}, \frac{1}{2}, \frac{1}{2} & \left.\frac{1}{4}\right), \frac{3}{2}
\end{array}\right) \\
& \frac{24}{\pi^{2}} F(2,3)=3 \log 2-\frac{1}{32}{ }_{4} F_{3}\left(\begin{array}{c|c}
\frac{3}{2}, \frac{3}{2}, 1,1 & \frac{1}{4} \\
2,2,2 & ,
\end{array}\right. \\
& \frac{15}{\pi^{2}} F(2,3)=\frac{1}{2} \log 18-\frac{1}{9}{ }_{4} F_{3}\left(\begin{array}{c|c}
\frac{3}{2}, \frac{3}{2}, 1,1 & \frac{8}{9} \\
2,2,2 & 9
\end{array}\right), \\
& \frac{9}{\pi^{2}} F(2,3)=\frac{1}{2} \log 2+{ }_{4} F_{3}\left(\begin{array}{c|c}
\frac{3}{2}, \frac{3}{2}, 1,1 \\
2,2,2
\end{array} \mid-8\right) \text {, }
\end{aligned}
$$




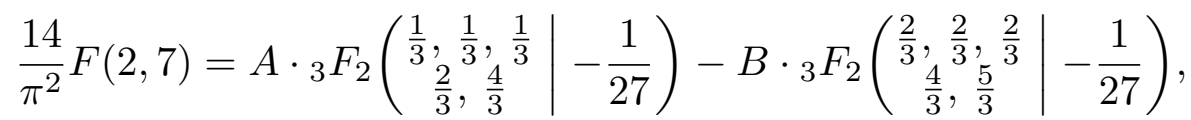

$$
\begin{aligned}
& \frac{49}{2 \pi^{2}} F(2,7)=\log 5-\frac{2}{125}{ }_{4} F_{3}\left(\begin{array}{c|c}
\frac{4}{3}, \frac{5}{3}, 1,1 & \frac{27}{125} \\
2,2,2 & ,
\end{array}\right. \\
& \frac{15}{4 \pi^{2}} F(3,5)=\frac{1}{4}{ }_{3} F_{2}\left(\begin{array}{c|c}
\frac{1}{2}, \frac{1}{2}, \frac{1}{2} & \frac{1}{16}, \frac{3}{2}
\end{array}\right), \\
& \frac{45}{2 \pi^{2}} F(3,5)=\log 5-\frac{2}{25}{ }_{4} F_{3}\left(\begin{array}{c|c}
\frac{3}{2}, \frac{3}{2}, 1,1 & \frac{16}{25} \\
2,2,2 & ,
\end{array}\right. \\
& \frac{165}{4 \pi^{2}} F(3,5)=4 \log 2-\frac{1}{128}{ }_{4} F_{3}\left(\begin{array}{c|c}
\frac{3}{2}, \frac{3}{2}, 1,1 & \frac{1}{16} \\
2,2,2 & 16
\end{array}\right)
\end{aligned}
$$

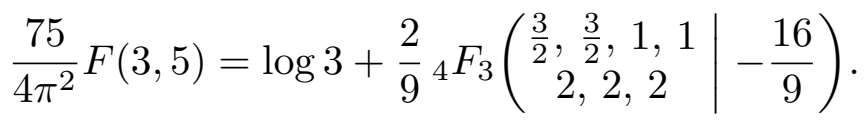

All these Boyd's evaluations corresponding to the entries in Table 3 are now rigorously established but this is far from exhausting the conjectures in Boyd's complete list [34], [35]. The 'lacunary' sums $F(1,1), F(1,2)$ and $F(1,3)$ are settled by F. Rodríguez-Villegas in [120]; A. Mellit has given a $K$-theoretic proof of the formulae for $F(2,7)$ in [98]; the relations for $F(1,5), F(2,3)$ and $F(3,5)$ are proved in our joint papers [124], [125] with M. Rogers.

The methods of [124], [125] are quite elementary in nature. One writes

$$
F(a, b, c, d)=-\int_{0}^{1} \eta(a \tau) \eta(b \tau) \eta(c \tau) \eta(d \tau) \frac{\log q}{q} \mathrm{~d} q, \quad q=e^{2 \pi i \tau}
$$

as

$$
F(a, b, c, d)=\int_{0}^{1} x(q) \log y(q) \mathrm{d} z(q)
$$

where $x(q), y(q)$ and $z(q)$ are modular functions on a congruence subgroup of $\mathrm{SL}_{2}(\mathbb{Z})$ (and this step can be usually achieved in several ways), and next express $x$ and $y$ as algebraic functions of $z$, that is, $x(q)=X(z(q))$ and $y(q)=Y(z(q))$. The substitution reduces $F(a, b, c, d)$ to a complicated integral of elementary functions; for example,

$$
F(2,3)=-\frac{\pi}{48} \int_{0}^{1 / 2} \frac{\sqrt{(1-2 p)(2-p)} \log \frac{p^{3}(2-p)}{1-2 p}}{\left(1-p^{2}\right) \sqrt{p}} \mathrm{~d} p .
$$

The final step is to reduce the elementary integral to Mahler measures. In order to accomplish this reduction we use properties of hypergeometric functions and elliptic functions. The machinery from [124], [125] allowed us to obtain different hypergeometric expressions for $F(1,1)$ and $F(1,3)$ as well as to deduce a new functional equation for the Mahler measure

$$
\mathrm{m}((1+x)(1+y)(x+y)-\alpha x y), \quad \alpha \in \mathbb{R} .
$$

All this reflects highly non-trivial identities between hypergeometric functions which do not possess 'purely hypergeometric' proofs. 
There are plenty of results and conjectures for $n$-variable Mahler measures with $n>2$, mostly motivated by $K$-theoretic considerations. Besides (101), Smyth established [33] that

$$
\mathrm{m}(1+x+y+z)=\frac{7 \zeta(3)}{2 \pi^{2}}
$$

and Rodriguez-Villegas further discovered numerically [56] that

$$
\begin{aligned}
\mathrm{m}(1+x+y+z+t) & \stackrel{?}{=}-\left(\frac{\sqrt{15}}{2 \pi}\right)^{5} \int_{0}^{1}\left(\eta^{3}(3 \tau) \eta^{3}(5 \tau)+\eta^{3}(\tau) \eta^{3}(15 \tau)\right) \frac{\log ^{3} q}{q} \mathrm{~d} q, \\
\mathrm{~m}(1+x+y+z+t+w) & \stackrel{?}{=}\left(\frac{3}{\pi^{2}}\right)^{3} \int_{0}^{1} \eta^{2}(\tau) \eta^{2}(2 \tau) \eta^{2}(3 \tau) \eta^{2}(6 \tau) \frac{\log ^{4} q}{q} \mathrm{~d} q .
\end{aligned}
$$

These evaluations are addressed in [122] and also in [31] in relation with short random walks on the plane.

\section{Bibliography}

[1] M. Aganagic, V. Bouchard, and A. Klemm, "Topological strings and (almost) modular forms", Comm. Math. Phys. 277:3 (2008), 771-819.

[2] G. Almkvist and J. Guillera, "Ramanujan-like series for $1 / \pi^{2}$ and string theory", Preprint at arXiv: 1009.5202 [math.NT], 2010.

[3] G. Almkvist, C. van Enckevort, D. van Straten, and W. Zudilin, "Tables of Calabi-Yau equations", Preprint at arXiv: math.AG/0507430, 2005-2010.

[4] G. Almkvist, D. van Straten, and W. Zudilin, "Apéry limits of differential equations of order 4 and 5", Modular Forms and String Duality, Fields Inst. Commun. Ser., vol. 54, Amer. Math. Soc. \& Fields Inst., Providence, RI 2008, pp. 105-123.

[5] G. Almkvist, D. van Straten, and W. Zudilin, "Generalizations of Clausen's formula and algebraic transformations of Calabi-Yau differential equations", Proc. Edinburgh Math. Soc. 54:2 (2011), 273-295.

[6] G. Almkvist and W. Zudilin, "Differential equations, mirror maps and zeta values", Mirror Symmetry V, AMS/IP Studies in Adv. Math., vol. 38, Amer. Math. Soc. \& International Press, Providence, RI 2006, pp. 481-515.

[7] Y. André, G-functions and geometry, Aspects Math., vol. E13, Friedr. Vieweg \& Sohn, Braunschweig 1989.

[8] G. E. Andrews, "Problems and prospects for basic hypergeometric functions", Theory and application of special functions, Proc. Advanced Sem., Math. Res. Center (Univ. Wisconsin, Madison, WI, 1975), Math. Res. Center, Univ. Wisconsin, vol. 35 (R. A. Askey, ed.), Academic Press, New York 1975, pp. 191-224.

[9] G. E. Andrews, "The well-poised thread: An organized chronicle of some amazing summations and their implications", Ramanujan J. 1:1 (1997), 7-23.

[10] G. E. Andrews, R. Askey, and R. Roy, Special functions, Encyclopedia Math. Appl., vol. 71, Cambridge Univ. Press, Cambridge 1999.

[11] G. E. Andrews and B. C. Berndt, Ramanujan's Lost Notebook. Part I, Springer-Verlag, New York 2005; Part II, Springer-Verlag, New York 2009.

[12] R. Apéry, "Irrationalité de $\zeta(2)$ et $\zeta(3)$ ", Journées arithmétiques de Luminy (Luminy, 1978), Astérisque, vol. 61, 1979, pp. 11-13. 
[13] W. N. Bailey, Generalized hypergeometric series, Cambridge Tracts in Math., vol. 32, Cambridge Univ. Press, Cambridge 1935; 2nd reprinted ed., Stechert-Hafner, New York-London 1964.

[14] A. Baker and J. Coates, "Fractional parts of powers of rationals", Math. Proc. Cambridge Philos. Soc. 77:2 (1975), 269-279.

[15] K. Ball et T. Rivoal, "Irrationalité d'une infinité de valeurs de la fonction zêta aux entiers impairs", Invent. Math. 146:1 (2001), 193-207.

[16] N. D. Baruah, B. C. Berndt, and H.H. Chan, "Ramanujan's series for $1 / \pi$ : a survey", Amer. Math. Monthly 116:7 (2009), 567-587.

[17] M. A. Bennett, "Fractional parts of powers of rational numbers", Math. Proc. Cambridge Philos. Soc. 114:2 (1993), 191-201.

[18] M. A. Bennett, "An ideal Waring problem with restricted summands", Acta Arith. 66:2 (1994), 125-132.

[19] B. C. Berndt, Ramanujan's notebooks. Part I, Springer-Verlag, New York 1985; Part II, Springer-Verlag, New York 1989; Part III, Springer-Verlag, New York 1991; Part IV, Springer-Verlag, New York 1994; Part V, Springer-Verlag, New York 1998.

[20] M. J. Bertin, "Mesure de Mahler d'une famille de polynômes", J. Reine Angew. Math. 569 (2004), 175-188.

[21] F. Beukers, "A note on the irrationality of $\zeta(2)$ and $\zeta(3)$ ", Bull. London Math. Soc. 11:3 (1979), 268-272.

[22] F. Beukers, "Fractional parts of powers of rationals", Math. Proc. Cambridge Philos. Soc. 90:1 (1981), 13-20.

[23] F. Beukers, "Irrationality of $\pi^{2}$, periods of an elliptic curve and $\Gamma_{1}(5)$ ", Diophantine approximations and transcendental numbers (Luminy, 1982), Progr. Math., vol. 31, Birkhäuser, Boston, MA 1983, pp. 47-66.

[24] F. Beukers, "Irrationality proofs using modular forms", Journées arithmétiques de Besançon" (Besançon, 1985), Astérisque, vol. 147-148, 1987, pp. 271-283.

[25] F. Beukers, "On Dwork's accessory parameter problem", Math. Z. 241:2 (2002), 425-444.

[26] F. Beukers and G. Heckman, "Monodromy for the hypergeometric function ${ }_{n} F_{n-1} "$, Invent. Math. 95:2 (1989), 325-354.

[27] J.-P. Bézivin, "Indépendence linéaire des valeurs des solutions transcendantes de certaines équations fonctionnelles", Manuscripta Math. 61:1 (1988), 103-129.

[28] J. W. Bober, "Factorial ratios, hypergeometric series, and a family of step functions", J. London Math. Soc. (2) 79 (2009), 422-444.

[29] M. Bogner, Differentielle Galoisgruppen und Transformationstheorie für Calabi-Yau-Operatoren vierter Ordnung, Diploma-Thesis, Institut für Mathematik, Johannes Gutenberg-Universität, Mainz 2008.

[30] J. M. Borwein and P. B. Borwein, Pi and the AGM; A study in analytic number theory and computational complexity, Wiley, New York 1987.

[31] J. M. Borwein, A. Straub, J. Wan, and W. Zudilin, "Densities of short uniform random walks", Canad. J. Math. (to appear); Preprint at arXiv: 1103.2995 [math.CA], 2011.

[32] P. Borwein, "On the irrationality of $\sum \frac{1}{q^{n}+r} "$, J. Number Theory 37 (1991), 253-259.

[33] D. W. Boyd, "Speculations concerning the range of Mahler's measure", Canad. Math. Bull. 24:4 (1981), 453-469.

[34] D. W. Boyd, "Mahler's measure and special values of $L$-functions", Experiment. Math. 7:1 (1998), 37-82. 
[35] D. W. Boyd, "Mahler's measure and invariants of hyperbolic manifolds", Number theory for the millennium, I, A K Peters, Natick, MA 2002, pp. 127-143.

[36] F. Brunault, "Version explicite du théorème de Beilinson pour la courbe modulaire $X_{1}(N)$ ", C. R. Math. Acad. Sci. Paris 343:8 (2006), 505-510.

[37] P. Bundschuh and K. Väänänen, "Arithmetical investigations of a certain infinite product", Compositio Math. 91 (1994), 175-199.

[38] P. Bundschuh and K. Väänänen, "Linear independance of $q$-analogues of certain classical constants", Result. Math. 47 (2005), 33-44.

[39] P. Bundschuh and W. Zudilin, "Rational approximations to a $q$-analogue of $\pi$ and some other $q$-series", Diophantine Approximation, Dev. Math., vol. 16, Springer-Verlag, Vienna 2008, pp. 123-139.

[40] P. Candelas, X. C. de la Ossa, P. S. Green, and L. Parkes, "A pair of Calabi-Yau manifolds as an exactly soluble superconformal theory", Nuclear Phys. B 359:1 (1991), 21-74.

[41] Y.-H. Chen, Y. Yang, and N. Yui, "Monodromy of Picard-Fuchs differential equations for Calabi-Yau threefolds", with an appendix by C. Erdenberger, J. Reine Angew. Math. 616 (2008), 167-203.

[42] G. V. Chudnovsky, "On the method of Thue-Siegel", Ann. of Math. (2) 117:2 (1983), 325-382.

[43] D. V. Chudnovsky and G. V. Chudnovsky, "Approximations and complex multiplication according to Ramanujan", Ramanujan revisited (Urbana-Champaign, IL, 1987), Academic Press, Boston, MA 1988, pp. 375-472.

[44] J. Cullen, "Pi formula", Preprint (December 2010).

[45] E. Delaygue, "Critère pour l'intégralité des coefficients de Taylor des applications miroir", J. Reine Angew. Math. (to appear); Preprint at arXiv: 0912.3776 [math.NT], 2009.

[46] F. Delmer and J.-M. Deshouillers, "The computation of $g(k)$ in Waring's problem", Math. Comp. 54 (1990), 885-893.

[47] C. Deninger, "Deligne periods of mixed motives, $K$-theory and the entropy of certain $\mathbb{Z}^{n}$-actions", J. Amer. Math. Soc. 10:2 (1997), 259-281.

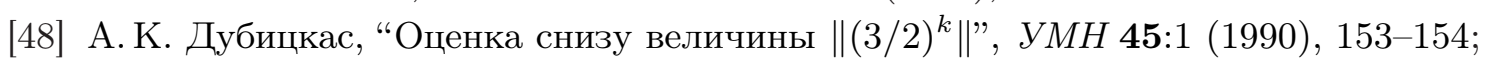
English transl., A. K. Dubitskas [A. Dubickas], "A lower bound on the value of $\left\|(3 / 2)^{k}\right\| "$, Russian Math. Surveys 45:4 (1990), 163-164.

[49] D. Duverney, "Irrationalité d'un $q$-analogue de $\zeta(2)$ ", C. R. Acad. Sci. Paris Sér. I Math. 321:10 (1995), 1287-1289.

[50] R. Dvornicich and C. Viola, "Some remarks on Beukers' integrals", Number theory, Vol. II (Budapest, 1987), Colloq. Math. Soc. János Bolyai, vol. 51, North-Holland, Amsterdam 1987, pp. 637-657.

[51] S. B. Ekhad and D. Zeilberger, "A WZ proof of Ramanujan's formula for $\pi$ ", Geometry, Analysis, and Mechanics (J. M. Rassias, ed.), World Sci. Publ., River Edge, NJ 1994, pp. 107-108.

[52] P. Erdös, "On arithmetical properties of Lambert series", J. Indiana Math. Soc. (N.S.) 12 (1948), 63-66.

[53] L. Euler, "Variae observationes circa series infinitas", Comm. Acad. Sci. Imp. Petropol. 9 (1737), 160-188; Reprint:, Opera Omnia Ser. I, vol. 14, Teubner, Berlin 1925, pp. 216-245.

[54] L. Euler, "Meditationes circa singulare serierum genus", Novi Comm. Acad. Sci. Petropol. 20 (1775), 140-186; Reprint, Opera Omnia Ser. I, vol. 15, Teubner, Berlin 1927, pp. 217-267. 
[55] S. R. Finch, Mathematical constants, Encyclopedia Math. Appl., vol. 94, Cambridge University Press, Cambridge 2003.

[56] S. R. Finch, "Modular forms on $\mathrm{SL}_{2}(\mathbb{Z})$ ", Preprint at http://algo.inria.fr/ csolve/frs.pdf, 2005.

[57] S. Fischler, "Irrationalité de valeurs de zêta [d'après Apéry, Rivoal, ...]", Astérisque 294 (2004), 27-62.

[58] S. Fischler and W. Zudilin, "A refinement of Nesterenko's linear independence criterion with applications to zeta values", Math. Ann. 347:4 (2010), 739-763.

[59] P. J. Forrester and M. L. Glasser, "Some new lattice sums including an exact result for the electrostatic potential within the NaCl lattice", J. Phys. A Math. Gen. 15 (1982), 911-914.

[60] J. Franel, "On a question of Laisant", L'intermédiaire des mathématiciens, vol. 1, Gauthier-Villars, Paris 1894, pp. 45-47; "On a question of J. Franel", L'intermédiaire des mathématiciens, vol. 2, Gauthier-Villars, Paris 1895, pp. 33-35.

[61] G. Gasper and M. Rahman, Basic hypergeometric series, 2nd ed., Encyclopedia Math. Appl., vol. 96, Cambridge Univ. Press, Cambridge 2004.

[62] А. О. Гельфонд, Исчисление конечных разностей, 3-е изд., Наука, М. 1967; English transl., A. O. Gel'fond, Calculus of finite differences, Intern. Monographs Adv. Math. Phys., Hindustan Publishing Corp., Delhi 1971.

[63] M. L. Glasser, "Evaluation of lattice sums. IV. A five-dimensional sum", J. Phys. A Math. Gen. 16 (1975), 1237-1238.

[64] R. L. Graham, D. E. Knuth, and O. Patashnik, Concrete mathematics. A foundation for computer science, 2nd ed., Addison-Wesley, Reading, MA 1994.

[65] J. Guillera, "Some binomial series obtained by the WZ-method", Adv. in Appl. Math. 29:4 (2002), 599-603.

[66] J. Guillera, "About a new kind of Ramanujan-type series", Experiment. Math. 12:4 (2003), 507-510.

[67] J. Guillera, "Hypergeometric identities for 10 extended Ramanujan-type series", Ramanujan J. 15:2 (2008), 219-234.

[68] J. Guillera, "A new Ramanujan-like series for $1 / \pi^{2}$ ", Preprint at arXiv: 1003.1915 [math.NT], 2010.

[69] J. Guillera, "A matrix form of Ramanujan-type series fo $1 / \pi$ ", Gems in Experimental Mathematics, Contemp. Math., vol. 517 (T. Amdeberhan, L. A. Medina, and V.H. Moll, eds.), Amer. Math. Soc., Providence, RI 2010, pp. 189-206.

[70] J. Guillera, "Mosaic supercongruences of Ramanujan type", Experiment. Math. (to appear); Preprint at arXiv: 1007.2290 [math.NT], 2010.

[71] J. Guillera and W. Zudilin, "Divergent" Ramanujan-type supercongruences", Proc. Amer. Math. Soc. (to appear); Preprint at arXiv: 1004.4337 [math.NT], 2010.

[72] Л. А. Гутник, “Об иррациональности некоторых величин, содержащих $\zeta(3)$ ”, УMH 34:3 (1979), 190; Acta Arith. 42:3 (1983), 255-264; English transl., L. A. Gutnik, "On the irrationality of some quantities containing $\zeta(3)$ ", Eleven papers translated from the Russian, Amer. Math. Soc. Transl. Ser. 2, vol. 140, Amer. Math. Soc., Providence, RI 1988, pp. 45-55.

[73] A. J. Guttmann, "Lattice Green functions and Calabi-Yau differential equations", J. Phys. A Math. Theor. 42:23 (2009), 232001, 6 pp.

[74] L. Habsieger, "Explicit lower bounds for $\left\|(3 / 2)^{k}\right\|$ ", Acta Arith. 106 (2003), 299-309. 
[75] M. Hata, "Legendre type polynomials and irrationality measures", J. Reine Angew. Math. 407:1 (1990), 99-125.

[76] M. Hata, "A new irrationality measure for $\zeta(3)$ ”, Acta Arith. 92:1 (2000), 47-57.

[77] Т. Г. Хессами Пилеруд, "О линейной независимости векторов с полилогарифмическими координатами", Вестник МГУ. Сер. 1. Матем., мex., 1999, no. 6, 54-56; English transl., T. Hessami Pilehrood, "On the linear independence of vectors with polylogarithmic coordinates", Moscow Univ. Math. Bull. 54:6 (1999), 40-42.

[78] F. Jouhet et E. Mosaki, "Irrationalité aux entiers impairs positifs d'un $q$-analogue de la fonction zêta de Riemann", Intern. J. Number Theory 6:5 (2010), 959-988.

[79] C. Krattenthaler et T. Rivoal, Hypergéométrie et fonction zêta de Riemann, Mem. Amer. Math. Soc., vol. 186, Amer. Math. Soc., Providence, RI 2007, no. 875.

[80] C. Krattenthaler and T. Rivoal, "An identity of Andrews, multiple integrals, and very-well-poised hypergeometric series", Ramanujan J. 13 (2007), 203-219.

[81] C. Krattenthaler and T. Rivoal, "On a linear form for Catalan's constant", South East Asian J. Math. Sci. 6:2 (2008), 3-15.

[82] C. Krattenthaler and T. Rivoal, "On the integrality of the Taylor coefficients of mirror maps", Duke Math. J. 151:2 (2010), 175-218; "On the integrality of the Taylor coefficients of mirror maps. II", Commun. Number Theory Phys. 3:3 (2009), 555-591.

[83] C. Krattenthaler and T. Rivoal, "Multivariate $p$-adic formal congruences and integrality of Taylor coefficients of mirror maps", Théories galoisiennes et arithmétiques des équations différentielles, Séminaires et Congrès (L. Di Vizio and T. Rivoal, eds.), Soc. Math. France, Paris (to appear); Preprint at arXiv: 0804.3049 [math.NT], 2008.

[84] C. Krattenthaler, T. Rivoal et W. Zudilin, "Séries hypergéométriques basiques, $q$-analogues des valeurs de la fonction zêta et formes modulaires", Inst. Jussieu Math. J. 5:1 (2006), 53-79.

[85] J. Kubina and M. Wunderlich, "Extending Waring's conjecture up to 471600000", Math. Comp. 55 (1990), 815-820.

[86] N. Kurokawa and H. Ochiai, "Mahler measures via crystalization", Comment. Math. Univ. St. Pauli 54:2 (2005), 121-137.

[87] M. N. Lalín and M. D. Rogers, "Functional equations for Mahler measures of genus-one curves", Algebra Number Theory 1:1 (2007), 87-117.

[88] D. H. Lehmer, "Factorization of certain cyclotomic functions", Ann. of Math. (2) 34:3 (1933), 461-479.

[89] B. H. Lian and S.-T. Yau, "Differential equations from mirror symmetry", Surveys in differential geometry: differential geometry inspired by string theory, Surv.

Differ. Geom., vol. 5, Intern. Press, Boston, MA 1999, pp. 510-526.

[90] F. Lindemann, "Über die Zahl $\pi$ ", Math. Ann. 20:2 (1882), 213-225.

[91] H. Maass, Siegel's modular forms and Dirichlet series, Lecture Notes in Math., vol. 216, Springer-Verlag, Berlin-New York 1971.

[92] K. Mahler, "On the fractional parts of powers of real numbers", Mathematika 4:2 (1957), 122-124.

[93] K. Mahler, "An application of Jensen's formula to polynomials", Mathematika 7:2 (1960), 98-100.

[94] K. Mahler, "On algebraic differential equations satisfied by automorphic functions", J. Austral. Math. Soc. 10 (1969), 445-450.

[95] V. Maillot, Géométrie d'Arakelov des variétés toriques et fibrés en droites intégrables, Mém. Soc. Math. France (N.S.), vol. 80, Soc. Math. France, Paris 2000. 
[96] Y. Martin and K. Ono, "Eta-quotients and elliptic curves", Proc. Amer. Math Soc. 125:11 (1997), 3169-3176.

[97] T. Matalo-Aho, K. Väänänen, and W. Zudilin, "New irrationality measures for q-logarithms", Math. Comp. 75:254 (2006), 879-889.

[98] A. Mellit, "Elliptic dilogarithms and parallel lines", Preprint, 2009.

[99] H. Movasati, "Eisenstein type series for Calabi-Yau varieties", Preprint at arXiv: 1007.4181 [math.AG], 2010.

[100] Ю.В.Нестеренко, "О линейной независимости чисел", Вестник МГУ. Сер. 1. Матем., мех., 1985, no. 1, 46-54; English transl., Yu. V. Nesterenko, "Linear independence of numbers", Moscow Univ. Math. Bull. 40:1 (1985), 69-74.

[101] Ю.В. Нестеренко, "Некоторые замечания о Ц(3)", Матем. заметки 59:6 (1996), 865-880; English transl., Yu. V. Nesterenko, "Some remarks on $\zeta(3)$ ", Math. Notes 59:6 (1996), 625-636.

[102] Ю. В. Нестеренко, "Модулярные функции и вопросы трансцендентности,", Матем. сб. 187:9 (1996), 65-96; English transl., Yu. V. Nesterenko, "Modular functions and transcendence questions", Sb. Math. 187:9 (1996), 1319-1348.

[103] Е. М. Никишин, “Об иррациональности значений функций $F(x, s)$ ”, Матем. сб. 109:3 (1979), 410-417; English transl., E. M. Nikishin, "Irrationality of values of functions $F(x, s)$ ", Math. USSR Sb. 37:3 (1979), 381-388.

[104] K. Nishioka, "A conjecture of Mahler on automorphic functions", Arch. Math. 53:1 (1989), 46-51.

[105] M. Petkovšek, H. S. Wilf, and D. Zeilberger, $A=B$, A K Peters, Wellesley 1996.

[106] A. van der Poorten, "A proof that Euler missed... Apéry's proof of the irrationality of $\zeta(3)$ ", Math. Intelligencer 1:4 (1978/79), 195-203.

[107] K. Postelmans and W. Van Assche, "Irrationality of $\zeta_{q}(1)$ and $\zeta_{q}(2)$ ", J. Number Theory 126 (2007), 119-154.

[108] M. Prévost, "A new proof of the irrationality of $\zeta(3)$ using Padé approximants", $J$. Comput. Appl. Math. 67 (1996), 219-235.

[109] Ю.А. Пупырев, "О линейной и алгебраической независимости $q$-дзетазначений", Матем. заметки 78:4 (2005), 608-613; English transl., Yu. A. Pupyrev, "Linear and algebraic independence of $q$-zeta values", Math. Notes 78:4 (2005), 563-568.

[110] Ю. А. Пупырев, “Эффективизация нижней оценки для $\left\|(4 / 3)^{k}\right\| ”$, Матем. заметки 85:6 (2009), 927-935; English transl., Yu. A. Pupyrev, "Effectivization of a lower bound for $\left\|(4 / 3)^{k}\right\| "$, Math. Notes 85:6 (2009), 877-885.

[111] S. Ramanujan, "Modular equations and approximations to $\pi$ ", Quart. J. Math. Oxford Ser. (2) 45 (1914), 350-372; Reprinted:, G. H. Hardy, P. V. Sechu Aiyar, and B. M. Wilson (eds.), Collected papers of Srinivasa Ramanujan, Cambridge University Press \& Chelsea Publ., New York 1962, pp. 23-39.

[112] G. Rhin and C. Viola, "On a permutation group related to $\zeta(2)$ ", Acta Arith. 77:1 (1996), 23-56.

[113] G. Rhin and C. Viola, "The group structure for $\zeta(3)$ ", Acta Arith. 97:3 (2001), 269-293.

[114] O. Richter, "On transformation laws for theta functions", Rocky Mountain J. Math. 34:4 (2004), 1473-1481.

[115] B. Riemann, "Ueber die Anzahl der Primzahlen unter einer gegebenen Grösse", Monatsberichte der Berliner Akademie, 1859; Reprint, Gesammelte Werke, Teubner, Leipzig 1892.

[116] T. Rivoal, "La fonction zêta de Riemann prend une infinité de valeurs irrationnelles aux entiers impairs", C. R. Acad. Sci. Paris Sér. I Math. 331:4 (2000), 267-270. 
[117] T. Rivoal, Propriétés diophantiennes des valeurs de la fonction zêta de Riemann aux entiers impairs, Thèse de Doctorat, Univ. de Caen, Caen 2001.

[118] T. Rivoal, "Irrationalité d'au moins un des neuf nombres $\zeta(5), \zeta(7), \ldots, \zeta(21)$ ", Acta Arith. 103 (2002), 157-167.

[119] T. Rivoal and W. Zudilin, "Diophantine properties of numbers related to Catalan's constant", Math. Ann. 326:4 (2003), 705-721.

[120] F. Rodríguez-Villegas, "Modular Mahler measures I", Topics in number theory (University Park, PA, 1997), Math. Appl., vol. 467, Kluwer Acad. Publ., Dordrecht 1999, pp. 17-48.

[121] F. Rodríguez-Villegas, "Identities between Mahler measures", Number theory for the millennium, III, A K Peters, Natick, MA 2002, pp. 223-229.

[122] F. Rodríguez-Villegas, R. Toledano, and J. D. Vaaler, "Estimates for Mahler's measure of a linear form", Proc. Edinburgh Math. Soc. 47:2 (2004), 473-494.

[123] M. D. Rogers, "Hypergeometric formulas for lattice sums and Mahler measures", Intern. Math. Res. Not. (to appear).

[124] M. D. Rogers and W. Zudilin, "From $L$-series of elliptic curves to Mahler measures", Preprint at arXiv: 1012.3036 [math.NT], 2010.

[125] M. D. Rogers and W. Zudilin, "On the Mahler measure of $1+X+1 / X+Y+1 / Y$ ", Preprint at arXiv: 1102.1153 [math.NT], 2011.

[126] Е. А. Рухадзе, "Оценка снизу приближения $\ln 2$ рациональными числами", Вестник МГУ. Сер. 1. Матем., мех., 1987, no. 6, 25-29; English transl., E. A. Rukhadze, "Lower estimate for rational approximations of ln 2", Moscow Univ. Math. Bull. 42:6 (1987), 30-35.

[127] A. L. Schmidt, "Generalized q-Legendre polynomials", J. Comput. Appl. Math. 49:1-3 (1993), 243-249.

[128] A. L. Schmidt, "Legendre transforms and Apéry's sequences", J. Austral. Math. Soc. Ser. A 58:3 (1995), 358-375.

[129] А. Б. Шидловский, Трансцендентные числа, Наука, M. 1987; English transl., A. B. Shidlovskii, Transcendental numbers, de Gruyter Stud. Math., vol. 12, Walter de Gruyter \& Co., Berlin 1989.

[130] L. J. Slater, Generalized hypergeometric functions, Cambridge Univ. Press, Cambridge 1966.

[131] C. Smet and W. Van Assche, "Irrationality proof of a $q$-extension of $\zeta(2)$ using little q-Jacobi polynomials", Acta Arith. 138:2 (2009), 165-178.

[132] В. Н. Сорокин, "Аппроксимации Эрмита-Паде для систем Никишина и иррациональность $\zeta(3) "$, УМН 49:2 (1994), 167-168; English transl., V. N. Sorokin, "Hermite-Padé approximations for Nikishin systems and the irrationality of $\zeta(3)$ ", Russian Math. Surveys 49:2 (1994), 176-177.

[133] В.Н. Сорокин, "О мере трансцендентности числа $\pi^{2}$ ", Матем. сб. 187:12 (1996), 87-120; English transl., V. N. Sorokin, "A transcendence measure for $\pi^{2}$ ", Sb. Math. 187:12 (1996), 1819-1852.

[134] В.Н. Сорокин, "Теорема Апери", Вестник МГУ. Сер. 1. Матем., мех., 1998, no. 3, 48-53; English transl., V. N. Sorokin, "On Apéry's theorem", Moscow Univ. Math. Bull. 53:3 (1998), 48-52.

[135] В. Н. Сорокин, "Циклические графы и теорема Апери", УМН 57:3 (2002), 99-134; English transl., V. N. Sorokin, "Cyclic graphs and Apéry's theorem", Russian Math. Surveys 57:3 (2002), 535-571.

[136] V. Strehl, "Binomial identities - combinatorial and algorithmic aspects,", Discrete Math. 136:1-3 (1994), 309-346. 
[137] Z.-W. Sun, "Supercongruences and Euler sums", Preprint at arXiv: 1001.4453 [math.NT], 2010.

[138] Y. Tachiya, "Irrationality of certain Lambert series", Tokyo J. Math. 27:1 (2004), $75-85$.

[139] W. Van Assche, "Little $q$-Legendre polynomials and irrationality of certain Lambert series", Ramanujan J. 5 (2001), 295-310.

[140] О.Н. Василенко, "Некоторые формулы для значения дзета-функции Римана в целых точках", Теория чисел и ее приложения (Ташкент, 26-28 сентября 1990 г.), Тезисы докладов Республиканской научно-теоретической конференции, Ташкентский гос. пед. институт, Ташкент 1990, с. 27; English transl., O. N. Vasilenko, "Certain formulae for values of the Riemann zeta-function at integral points", Number theory and its applications (Tashkent, 26-28 September 1990), Proceedings of the science-theoretical conference, Tashkent State Pedagogical Inst., Tashkent 1990, pp. 27.

[141] Д. В. Васильев, "Некоторые формулы для дзета-функции в целых точках", Вестник МГУ. Сер. 1. Матем., мех., 1996, nо. 1, 81-84; English transl., D. V. Vasil'ev, "Some formulas for the Riemann zeta function at integer points", Moscow Univ. Math. Bull. 51:1 (1996), 41-43.

[142] D. V. Vasilyev, On small linear forms for the values of the Riemann zeta-function at odd points, Preprint № 1 (558), Nat. Acad. Sci. Belarus, Institute Math., Minsk 2001.

[143] R. C. Vaughan, The Hardy-Littlewood method, 2nd ed., Cambridge Tracts in Math., vol. 125, Cambridge Univ. Press, Cambridge 1997.

[144] C. Viola, "Birational transformations and values of the Riemann zeta-function", $J$. Théor. Nombres Bordeaux 15:2 (2003), 561-592.

[145] С. М. Воронин, А. А. Карацуба, Дзета-функция Римана, Физматлит, М. 1994; English transl., A. A. Karatsuba and S. M. Voronin, The Riemann zeta-function, de Gruyter Exp. Math., vol. 5, Walter de Gruyter \& Co., Berlin 1992.

[146] S. O. Warnaar and W. Zudilin, "A q-rious positivity", Aequat. Math. 81:1-2 (2011), $177-183$.

[147] F. J. W. Whipple, "A group of generalized hypergeometric series: relations between 120 allied series of the type $F[a, b, c ; d, e]$ ", Proc. London Math. Soc. (2) 23 (1925), 104-114.

[148] F. J. W. Whipple, "On well-poised series, generalized hypergeometric series having parameters in pairs, each pair with the same sum", Proc. London Math. Soc. (2) 24 (1926), 247-263.

[149] H. S. Wilf and D. Zeilberger, "An algorithmic proof theory for hypergeometric (ordinary and " $q$ ") multisum/integral identities", Invent. Math. 108:3 (1992), $575-633$.

[150] Y. Yang, "Apéry limits and special values of L-functions", J. Math. Anal. Appl. 343:1 (2008), 492-513.

[151] Y. Yang and W. Zudilin, "An $\mathrm{Sp}_{4}$ modularity of Picard-Fuchs differential equations for Calabi-Yau threefolds", with an appendix by V. Pasol, Gems in Experimental Mathematics, Contemp. Math., vol. 517 (T. Amdeberhan, L. A. Medina, and V. H. Moll, eds.), Amer. Math. Soc., Providence, RI 2010, pp. 381-413.

[152] M. Yoshida, Fuchsian differential equations. With special emphasis on the Gauss-Schwarz theory, Aspects Math., vol. E11, Friedr. Vieweg \& Sohn, Braunschweig 1987.

[153] D. Zagier, "The non-holomorphic embedding of $\mathfrak{H}$ into $\mathfrak{H}_{2}$ ", Unpublished note, 2008. 
[154] D. Zagier, "Integral solutions of Apéry-like recurrence equations", Groups and Symmetries: From Neolithic Scots to John McKay, CRM Proc. Lecture Notes, vol. 47, Amer. Math. Soc., Providence, RI 2009, pp. 349-366.

[155] D. Zeilberger, "Computerized deconstruction", Adv. Appl. Math. 31 (2003), $532-543$.

[156] С. А. Злобин, "Интегралы, представляемые в виде линейных форм от обобщенных полилогарифмов", Матем. заметки 71:5 (2002), 782-787; English transl., S. A. Zlobin, "Integrals expressible as linear forms in generalized polylogarithms", Math. Notes 71:5 (2002), 711-716.

[157] С. А. Злобин, "О некоторых интегральных тождествах", УМН 57:3 (2002), 153-154; English transl., S. A. Zlobin, "On some integral identities", Russian Math. Surveys 57:3 (2002), 617-618.

[158] С. А. Злобин, "Интегралы Рина", Матем. заметки 81:2 (2007), 226-239; English transl., S. A. Zlobin, "Rhin integrals", Math. Notes 81:2 (2007), 201-212.

[159] I. J. Zucker, "Madelung constants and lattice sums for hexagonal crystals", $J$. Phys. A Math. Gen. 24:4 (1991), 873-879.

[160] В. В. Зудилин, "Разностные уравнения и мера иррациональности чисел", Аналитическая теория чисел и приложения, Труды МИАН, 218, 1997, c. 165-178; English transl., W. Zudilin, "Difference equations and the irrationality measure of numbers", Proc. Steklov Inst. Math. 218 (1997), 160-174.

[161] W. Zudilin, "Number theory casting a look at the mirror", Preprint at arXiv: math.NT/0008237, 2000.

[162] В. В. Зудилин, "Одно из чисел $\zeta(5), \zeta(7), \zeta(9), \zeta(11)$ иррационально", УМН 56:4 (2001), 149-150; English transl., W. Zudilin, "One of the numbers $\zeta(5), \zeta(7)$, $\zeta(9), \zeta(11)$ is irrational", Russian Math. Surveys 56:4 (2001), 774-776.

[163] В. В. Зудилин, "Об иррациональности значений дзета-функции Римана", Изв. РАН. Серия матем. 66:3 (2002), 49-102; English transl., W. Zudilin, "Irrationality of values of the Riemann zeta function", Izv. Math. 66:3 (2002), 489-542.

[164] W. Zudilin, "Remarks on irrationality of q-harmonic series", Manuscripta Math. 107:4 (2002), 463-477.

[165] В. В. Зудилин, "О мере иррациональности $q$-аналога $\zeta(2)$ ", Матем. сб. 193:8 (2002), 49-70; English transl., W. Zudilin, "On the irrationality measure for a $q$-analogue of $\zeta(2)$ ", Sb. Math. 193:8 (2002), 1151-1172.

[166] В. В. Зудилин, "Совершенно уравновешенные гипергеометрические ряды и кратные интегралы”, УМН 57:4 (2002), 177-178; English transl., W. Zudilin, "Very well-poised hypergeometric series and multiple integrals", Russian Math. Surveys 57:4 (2002), 824-826.

[167] В. В. Зудилин, "О рекурсии третьего порядка типа Апери для $\zeta(5)$ ", Матем. заметки 72:5 (2002), 796-800; English transl., W. Zudilin, "A third-order Apéry-like recursion for $\zeta(5)$ ", Math. Notes 72:5 (2002), 733-737.

[168] В. В. Зудилин, "О диофантовых задачах для $q$-дзета-значений", Матем. заметки 72:6 (2002), 936-940; English transl., W. Zudilin, "Diophantine problems for q-zeta values", Math. Notes 72:6 (2002), 858-862.

[169] W. Zudilin, "The hypergeometric equation and Ramanujan functions", Ramanujan J. 7:4 (2003), 435-447.

[170] В. В. Зудилин, "О функциональной трансцендентности $q$-дзета-значений", Матем. заметки 73:4 (2003), 629-630; English transl., W. Zudilin, "On the functional transcendence of q-zeta values", Math. Notes 73:4 (2003), 588-589.

[171] W. Zudilin, "Well-poised hypergeometric service for diophantine problems of zeta values", J. Théor. Nombres Bordeaux 15:2 (2003), 593-626. 
[172] W. Zudilin, "Heine's basic transform and a permutation group for $q$-harmonic series", Acta Arith. 111:2 (2004), 153-164.

[173] W. Zudilin, "Arithmetic of linear forms involving odd zeta values", J. Théor. Nombres Bordeaux 16:1 (2004), 251-291.

[174] W. Zudilin, "Well-poised hypergeometric transformations of Euler-type multiple integrals", J. London Math. Soc. 70:1 (2004), 215-230.

[175] В. В. Зудилин, "О биномиальных суммах, связанных с рациональными приближениями к $\zeta(4)$ ", Матем. заметки 75:4 (2004), 637-640; English transl., W. Zudilin, "Binomial sums related to rational approximations to $\zeta(4)$ ", Math. Notes 75:4 (2004), 594-597.

[176] W. Zudilin, "On a combinatorial problem of Asmus Schmidt", Electron. J. Combin. 11:1 (2004), \#R22, 8 pages.

[177] В. В. Зудилин, "Об обратном преобразовании Лежандра одного семейства последовательностей”, Матем. заметки 76:2 (2004), 300-303; English transl., W. Zudilin, "The inverse Legendre transform of a certain family of sequences", Math. Notes 76:2 (2004), 276-279.

[178] W. Zudilin, "Approximations to $q$-logarithms and $q$-dilogarithms, with applications to q-zeta values", Труды по теории чисел, Зап. научн. сем. ПОМИ, 322, 2005, с. 107-124; Reprinted:, J. Math. Sci. (N. Y.) 137:2 (2006), $4673-4683$.

[179] W. Zudilin, "A new lower bound for $\left\|(3 / 2)^{k}\right\|$ ", J. Théor. Nombres Bordeaux 19:1 (2007), 313-325.

[180] W. Zudilin, "Approximations to -, di- and tri-logarithms", J. Comput. Appl. Math. 202:2 (2007), 450-459.

[181] W. Zudilin, "Ramanujan-type formulae for 1/ $\pi$ : A second wind?", Modular Forms and String Duality, Fields Inst. Commun. Ser., vol. 54, Amer. Math. Soc. \& Fields Inst., Providence, RI 2008, pp. 179-188.

[182] W. Zudilin, "Apéry's theorem. Thirty years after", Intern. J. Math. Computer Sci. 4:1 (2009), 9-19.

[183] W. Zudilin, "Ramanujan-type supercongruences", J. Number Theory 129:8 (2009), $1848-1857$.

\section{W. Zudilin}

School of Mathematical and Physical Sciences, University of Newcastle, Callaghan, Australia

E-mail: wadim.zudilin@newcastle.edu.au
Received 18/FEB/11 Translated by THE AUTHOR 